\title{
Magnetic energy fluxes in sub-Alfvénic planet star and moon planet interactions ${ }^{\star}$
}

\author{
J. Saur ${ }^{1}$, T. Grambusch ${ }^{1,2}$, S. Duling ${ }^{1}$, F. M. Neubauer ${ }^{1}$, and S. Simon ${ }^{1}$ \\ ${ }^{1}$ Institut für Geophysik und Meteorologie, Universität zu Köln, Cologne, Germany \\ e-mail: [saur;sduling; neubauer; simon]@geo.uni-koeln.de \\ 2 1. Physikalisches Institut, Universität zu Köln, Cologne, Germany \\ e-mail: grambusch@ph1.uni-koeln.de
}

Received 29 September 2011 / Accepted 2 January 2013

\section{ABSTRACT}

Context. Electromagnetic coupling of planetary moons with their host planets is well observed in our solar system. Similar couplings of extrasolar planets with their central stars have been studied observationally on an individual as well as on a statistical basis. Aims. We aim to model and to better understand the energetics of planet star and moon planet interactions on an individual and as well as on a statistical basis.

Methods. We derived analytic expressions for the Poynting flux communicating magnetic field energy from the planetary obstacle to the central body for sub-Alfvénic interaction. We additionally present simplified, readily useable approximations for the total Poynting flux for small Alfvén Mach numbers. These energy fluxes were calculated near the obstacles and thus likely present upper limits for the fluxes arriving at the central body. We applied these expressions to satellites of our solar system and to HD $179949 \mathrm{~b}$. We also performed a statistical analysis for 850 extrasolar planets.

Results. Our derived Poynting fluxes compare well with the energetics and luminosities of the satellites' footprints observed at Jupiter and Saturn. We find that 295 of 850 extrasolar planets are possibly subject to sub-Alfvénic plasma interactions with their stellar winds, but only 258 can magnetically connect to their central stars due to the orientations of the associated Alfvén wings. The total energy fluxes in the magnetic coupling of extrasolar planets vary by many orders of magnitude and can reach values larger than $10^{19} \mathrm{~W}$. Our calculated energy fluxes generated at HD 179949 b can only explain the observed energy fluxes for exotic planetary and stellar magnetic field properties. In this case, additional energy sources triggered by the Alfvén wave energy launched at the extrasolar planet might be necessary. We provide a list of extrasolar planets where we expect planet star coupling to exhibit the largest energy fluxes. As supplementary information we also attach a table of the modeled stellar wind plasma properties and possible Poynting fluxes near all 850 extrasolar planets included in our study.

Conclusions. The orders of magnitude variations in the values for the total Poynting fluxes even for close-in extrasolar planets provide a natural explanation why planet star coupling might have been only observable on an individual basis but not on a statistical basis.

Key words. planet-star interactions - planets and satellites: general - planets and satellites: magnetic fields

\section{Introduction}

Planetary bodies throughout the universe are commonly embedded in a flow of magnetized plasma. These bodies are thereby obstacles to the flow and interact with their surrounding plasma. Among the different types of waves excited in these interactions, the Alfvén mode is particularly important because it can transport energy and momentum along the local background magnetic field with very little dispersion over large distances. An interesting case of this interaction occurs if the relative velocity $v_{0}$ between the plasma and the obstacle is smaller than the Alfvén velocity $v_{\mathrm{A}}$, i.e. the Alfvén Mach number $M_{\mathrm{A}}=v_{0} / v_{\mathrm{A}}$ is smaller than 1 . Then a necessary condition is met that the Alfvén mode can carry energy and momentum in the upstream direction of the flow.

Sub-Alfvénic plasma interaction $\left(M_{\mathrm{A}}<1\right)$ is well known in our solar system. It has been observed and studied for artificial satellites in the Earth's magnetosphere (Drell et al. 1965). It is also common in the outer solar system, where the planetary

* Estimated plasma parameters and their associated Poynting fluxes are only available at the CDS via anonymous ftp to

cdsarc.u-strasbg.fr (130.79.128.5) or via

http://cdsarc.u-strasbg.fr/viz-bin/qcat?]/A+A/552/A119 satellites are often close enough to their parent planets such that their orbits are within the planets' magnetospheres. In these cases the relative velocities between the planetary satellites and the magnetospheric plasma are often small enough for the plasma interaction to be sub-Alfvénic. Historically, the interaction of Io with Jupiter's magnetosphere played the leading role in advancing, both observationally and theoretically, our understanding of sub-Alfvénic plasma interaction. Io's interaction has been observed through its control of Jupiter's radio waves (e.g., Bigg 1964; Zarka 1998), by in-situ measurements of the Voyager and Galileo spacecraft (e.g., Acuña et al. 1981; Kivelson et al. 1996b; Frank et al. 1996), and subsequently as Io's footprints in Jupiter's atmosphere (Connerney et al. 1993; Prangé et al. 1996; Clarke et al. 1996; Bonfond et al. 2008; Wannawichian et al. 2010; Bonfond 2012; Bonfond et al. 2013). In conjunction with the observational progress, the electrodynamic coupling between Io and Jupiter has been extensively studied theoretically and numerically as well (e.g., Piddington \& Drake 1968; Goldreich \& Lynden-Bell 1969; Neubauer 1980; Goertz 1980; Wright \& Schwartz 1989; Jacobsen et al. 2007, 2010). Next to Io, sub-Alfvénic satellite interactions with significant energy exchanges have also been observed at Jupiter's large satellites Europa, Ganymede and Callisto (Kivelson et al. 2004) 
and imprints of the interaction in Jupiter's atmosphere in form of auroral footprints have been observed for Europa and Ganymede (Clarke et al. 2002; Grodent et al. 2006, 2009; Bonfond 2012). Hints for a Callisto footprint have been reported by Clarke et al. (2011). Recently, Cassini spacecraft observations identified a significant sub-Alfvénic interaction at Saturn's satellite Enceladus generated by geyser activity near its south pole (Dougherty et al. 2006; Tokar et al. 2006; Khurana et al. 2007; Saur et al. 2007, 2008; Kriegel et al. 2009; 2011; Jia et al. 2010; Simon et al. 2011a). The Alfvén waves launched near Enceladus also generate footprints in Saturn's upper atmosphere as recently discovered (Pryor et al. 2011).

In our own solar system, all planets are sufficiently far away from the sun such that the relative velocity between the solar wind and the planets is super-Alfvénic $\left(M_{\mathrm{A}}>1\right)$ and superfast nearly all the time, i.e. the relative plasma velocity is larger than the group velocity of the fast magneto-sonic mode. In our solar system $M_{\mathrm{A}}=1$ occurs on average around $0.08 \mathrm{AU}$ and Mercury's perihelion is near 0.31 AU. Chané et al. (2012) recently reported an exceptional period where the solar wind upstream from Earth was sub-Alfvénic for a time period of four hours. During that time period the Earth lost its bow shock and developed Alfvén wings. The Alfvén wings were, however, not able to connect to our sun because the sub-Alfvénic period lasted not long enough. Many of the extrasolar planets discovered so far orbit their central stars within close distances, i.e. less than $0.1 \mathrm{AU}$. At close radial distance, the stellar wind likely often has not reached a flow speed which exceeds the Alfvén velocity. Thus the interaction is sub-Alfvénic and Alfvén waves generated by the interaction can travel upstream and transport energy to the central star when the orientation of the stellar wind magnetic field is favorable (as described in detail in this work). Similar sub-Alfvénic interactions occur in our solar system between planetary satellites and their central planets. The resulting sub-Alfvénic plasma interaction does not generate a bow-shock, but an Alfvén wing structure in the satellites/planets plasma environment. The interaction of extrasolar planets with their central star is commonly called star-planet interaction (SPI) in the literature (e.g., Shkolnik et al. 2003).

Observational evidence for such a magnetic extrasolar planet star coupling comes from measurements of enhanced stellar Ca emission correlated with the orbital periods of close in extrasolar planets by Shkolnik et al. (2003, 2005, 2008). In particular for HD 179949, the synchronicity of the Ca emission with the orbital period is visible in four out of six epochs (Shkolnik et al. 2008). For this star, Shkolnik et al. (2005) estimate a chromospheric excess energy flux of $\sim 10^{20}$ Watt, i.e. the same order of magnitude as a typical flare.

Next to individual studies, SPI is also investigated on a statistical basis. Scharf (2010) presents an analysis of X-ray fluxes from extrasolar planet harboring stars and argues that stars with extrasolar planets closer than 0.15 AU show a correlation of $\mathrm{X}$-ray flux with the mass of the extrasolar planets, while extrasolar planets at larger distances show no correlation. Poppenhaeger et al. (2010) also provide a statistical analysis of X-ray fluxes from a sample of 72 stars, which host extrasolar planets, but arrive at a different conclusion compared to Scharf (2010). The authors show that there are no significant correlations of the normalized X-ray flux with planetary mass or semi-major axis. Thus Poppenhaeger et al. (2010) see no statistical evidence of SPI even though SPI might still be observable for some individual targets. In their most recent study Poppenhaeger \& Schmitt (2011) argue that the correlation derived in Scharf (2010) is caused by selection effects and does not trace possible planet induced phenomena in stellar coronae. In another study in the $v$ Andromedae system, Poppenhaeger et al. (2011) also find no evidence in X-rays or in the optical that can be identified being due to extrasolar planets.

Theoretical aspects of the plasma interaction at extrasolar planets have been addressed by a series of authors. Cuntz et al. (2000) estimate with simplified expressions the strengths of tidal and magnetic planet star couplings for 12 planet star systems. Ip et al. (2004) numerically model the SPI of close-in extrasolar planets assuming that the planets possess a magnetic field and therefore also a planetary magnetosphere. Preusse et al. (2005, 2006,2007 ) also numerically model the sub-Alfvénic interaction of hot Jupiter's with the stellar winds and the phase difference generated by the finite propagation time of the Alfvén waves to the central star. Further numerical simulations were performed by Lipatov et al. (2005) and Cohen et al. (2009). Kopp et al. (2011) numerically investigate SPI for magnetized and nonmagnetized planets and conclude that the mere existence of SPI is no evidence that a planet possesses an intrinsic magnetic field. An example for this argument are the moons Io (nonmagnetized) and Ganymede (magnetized), which both couple to Jupiter. In the case of Io, the existence of an atmosphere and ionosphere is sufficient to cause a strong interaction with the magnetospheric plasma of Jupiter and to generate powerful Alfvén wings.

Grießmeier et al. (2004, 2005, 2007) investigate SPI with particular emphasis on the radio emission from extrasolar planets and their detectability from Earth depending on various parameters such as stellar wind properties. Zarka et al. (2001), Zarka (2006, 2007), and Hess \& Zarka (2011) also investigate the plasma interaction of extrasolar planets with their parent star and their associated putative radio emission. Li et al. (1998), Willes \& Wu (2004, 2005), and Hess \& Zarka (2011) study the possible electromagnetic coupling of extrasolar planets around white dwarfs and their effects on radio emission and orbital evolution. In several of these studies expressions for the Poynting flux convected onto the planetary obstacle and the energy dissipated in the planets' ionosphere/magnetosphere are calculated.

Lanza $(2008,2009)$ also investigate magnetic star-planet interaction with theoretical models. Lanza (2008) discusses the energy budget under the assumption that the planets trigger a release of the energy of the coronal fields by decreasing their relative helicity. The observed intermittent character of the starplanet interaction by Shkolnik et al. (2008) is explained by a topological change in the stellar coronal field, induced by a variation in its relative helicity.

Even though there exists no observational evidence for extrasolar planets to possess an intrinsic magnetic field, yet, it is still often assumed to be the case (e.g., Christensen et al. 2009) as many of the extrasolar planets are assumed to be similar in structure as the outer planets of our solar system, which all possess dynamo fields. In this case the sub-Alfvénic interaction of a stellar wind with an extrasolar planet would likely be similar to Ganymede's sub-Alfvénic interaction with the plasma of Jupiter's magnetosphere as Ganymede is the only known planetary satellite with an intrinsic dynamo field (Kivelson et al. 1996a).

The aim of this work is to study the electromagnetic energy fluxes, i.e. Poynting fluxes, radiated away from satellites or extra solar planets in sub-Alfvénic interaction. Previous studies used the Poynting flux onto the satellite/plasma based on constant magnetic field and plasma velocities as a proxy for the energy fluxes radiated away from the satellites/planets. In our work we derive explicit expressions for the Poynting flux including the nonlinear magnetic field and plasma velocities in the Alfvén 
waves generated by the interaction. We additionally present simplified, readily useable approximations for the Poynting flux for small Mach numbers. Our calculated energy fluxes are benchmarked based on well observed sub-Alfvénic interactions in our solar system, i.e. Io, Europa, Ganymede, and Enceladus. Then we apply our model to the 850 extrasolar planets discovered until 2012 November 14 to perform a statistical study. We also look individually at HD $179949 \mathrm{~b}$ and compare our results with the observations by Shkolnik et al. (2003, 2005, 2008). We determine for each extrasolar planet whether sub-Alfvénic interaction is to be expected, whether the Alfvén waves can travel upstream, and we then subsequently estimate the energy flux within the Alfvén wings, which are generated at each extrasolar planet. We particularly investigate how geometrical and plasma properties, such as the angle between the local magnetic field and the plasma flow or the orientation of a possible dipole moment of the planetary body affect the values of the Poynting flux and its ability to travel upstream.

The remainder of the work is structured in the following way: in Sect. 2.1 we derive expressions for the total Poynting flux, whose properties and dependencies are discussed in Sects. 2.2 and 2.3. The derived fluxes are then benchmarked in our solar system (Sect. 3) and finally applied to an ensemble of 850 known extrasolar planets, including HD 179949 b (Sect. 4).

\section{Model for the Poynting flux within the Alfvén wings}

A planetary obstacle in a flow of magnetized plasma modifies the electric and magnetic field within the vicinity of the planetary body and generates waves, which radiate electromagnetic, mechanical and thermal energy away from the obstacle.

\subsection{Calculation of Poynting flux in sub-Alfvénic plasma interaction}

Poynting's theorem for the evolution of the electromagnetic field energy reads

$\frac{\partial}{\partial t}\left(\frac{B^{2}}{2 \mu_{0}}+\frac{E^{2} \epsilon_{0}}{2}\right)+\nabla \cdot \boldsymbol{S}=-\boldsymbol{j} \cdot \boldsymbol{E}$

with the electric field $\boldsymbol{E}$, the magnetic induction $\boldsymbol{B}$, the magnetic and electric permeabilities of free space $\mu_{0}$ and $\epsilon_{0}$, respectively, the electric current density $\boldsymbol{j}$, and the Poynting flux

$\boldsymbol{S}=\boldsymbol{E} \times \boldsymbol{B} / \mu_{0}$.

In the ideal magneto-hydrodynamic (MHD) approximation, i.e. $\boldsymbol{E}=-\boldsymbol{v} \times \boldsymbol{B}$, the Poynting flux can equivalently be written as

$S=\left(\frac{B^{2}}{\mu_{0}}\right) \boldsymbol{v}_{\perp}$,

i.e. the Poynting flux describes the transport of magnetic enthalpy, which is bodily carried by the plasma velocity $\boldsymbol{v}_{\perp}$ perpendicular to the magnetic field.

\subsubsection{Sub-Alfvénic interaction and Alfvén wings}

If a plasma with magnetic field $\boldsymbol{B}_{0}$ and mass density $\rho$ convects with a relative velocity $\boldsymbol{v}_{0}$ past a planetary body, an observer in the rest frame of the planetary body sees a motional electric field $\boldsymbol{E}_{0}=-\boldsymbol{v}_{0} \times \boldsymbol{B}_{0}$ with $E_{0}=\cos \Theta v_{0} B_{0}$ assuming frozen-in-field conditions. Here the angle $\Theta$ describes the deviation of the flow direction from being perpendicular to

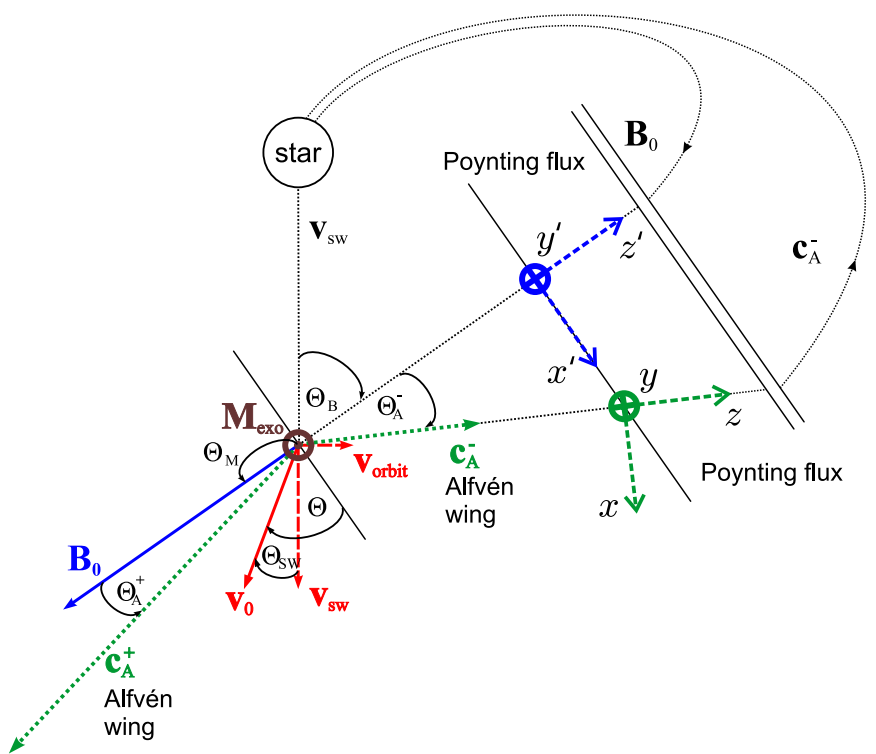

Fig. 1. Geometrical properties of the interaction of a planetary body with its surrounding magnetized plasma with $\boldsymbol{B}_{0}$ : unperturbed stellar wind magnetic field, $\boldsymbol{v}_{\mathrm{sw}}$ : stellar wind velocity, $\boldsymbol{v}_{\text {orbit }}$ : orbital velocity of planet, $\boldsymbol{v}_{0}$ : relative velocity between planet and stellar wind plasma, $\boldsymbol{M}_{\mathrm{exo}}$ : magnetic moment of planet (in this figure it points out of the displayed plane), $\boldsymbol{c}_{\mathrm{A}}^{+}$: direction of Alfvén wing in parallel direction of $\boldsymbol{B}_{0}$, $\boldsymbol{c}_{\mathrm{A}}^{-}$: direction of Alfvén wing in anti-parallel direction of $\boldsymbol{B}_{0}, \Theta$ : angle between $\boldsymbol{v}_{0}$ and normal to $\boldsymbol{B}_{0}, \Theta_{\mathrm{A}}^{ \pm}$: angles between both Alfvén wings and $\boldsymbol{B}_{0}$, and $\Theta_{M}$ : angle between $\boldsymbol{B}_{0}$ and planetary magnetic moment $\boldsymbol{M}_{\text {exo }}$. The Poynting flux is calculated through a plane perpendicular to $\boldsymbol{B}_{0}$. Note, the properties are displayed in the rest frame of the obstacle, besides the orbital and stellar wind velocity (which have been added for clarity in an inertial rest frame).

the magnetic field with $0 \leq \Theta \leq \pi$ (see Fig. 1). The planetary body represents an obstacle to the flow and generates Alfvén waves with group velocities $\boldsymbol{v}_{\mathrm{A}}= \pm \boldsymbol{B}_{0} / \sqrt{\rho \mu_{0}}$ in the rest frame of the plasma. If the Alfvén Mach number $M_{\mathrm{A}}=$ $v_{0} / v_{\mathrm{A}}<1$, then a necessary condition is met that the Alfvén waves can propagate upstream of the flow. In the sub-Alfvénic case, two standing Alfvén waves, also called Alfvén wings (Neubauer 1980) are generated (see Fig. 1). Sufficiently far away from the obstacle such that the slow mode and fast mode have insignificant wave amplitudes, the Elsasser variables or Alfvén characteristics

$c_{\mathrm{A}}^{ \pm}=\boldsymbol{v} \pm \boldsymbol{v}_{\mathrm{A}}$

are conserved quantities in each wing when the stellar wind plasma is sufficiently smooth and the plasma $\beta$ sufficiently low (Elsässer 1950; Neubauer 1980).

In this work we calculate the energy fluxes radiated away from the obstacles. We assume that in the vicinity of the extrasolar planets (or planetary moons) the incoming stellar wind properties (or magnetospheric plasma properties) can be considered spatially homogeneous on the scales of the local plasma interaction. The energy fluxes generated at the planets (moons) are calculated through a plane which is chosen to be sufficiently far away from the planets (or moons) such that other waves modes than the shear Alfvén modes do not play a role any more. But the location of the plane is still chosen close enough to the planet (or moon) such that the stellar wind properties can still be considered spatially homogeneous and, e.g. the bend of the Parker spiral or the curvature of the magnetospheric fields do not need to be considered. Typical distances of the plane from the planetary 
obstacles are several times the diameter of the obstacle, whose sizes will be discussed further below.

For the overall stellar wind flow, we assume that the velocity $\boldsymbol{v}_{\mathrm{sw}}$ is strictly radially away from the star. We also assume without restriction of generality that the magnetic field direction of the stellar wind points away from the star. The planets move in orbital direction with Kepler velocity $\boldsymbol{v}_{\text {orbit }}$, which leads to a relative velocity between the planet and the stellar wind of

$\boldsymbol{v}_{0}=\boldsymbol{v}_{\mathrm{sw}}-\boldsymbol{v}_{\text {orbit }}$.

One of the two Alfvén wings generated in the interaction always points away from the star. With our choice of magnetic field orientations of $\boldsymbol{B}_{0}$, this is the $\boldsymbol{c}_{\mathrm{A}}^{+}$-wing. We therefore focus in this study on the $\boldsymbol{c}_{\mathrm{A}}^{-}$-wing (see Fig. 1). The expressions in the remainder of this work thus hold for the $\boldsymbol{c}_{\mathrm{A}}^{-}$-wing, but could similarly be derived for the $c_{\mathrm{A}}^{+}$-wing. In the remainder of the manuscript we drop for simplicity of notation the superscript "-, on the quantities describing the $\boldsymbol{c}_{\mathrm{A}}^{-}$-wing.

For the moon-magnetosphere interaction, the geometry is different. The plasma flow $\boldsymbol{v}_{\mathrm{p}}$ is mostly in the orbital direction of the moon and the magnetic field is nearly perpendicular to the flow. The relative velocity $\boldsymbol{v}_{0}$ thus can be written

$$
\begin{aligned}
\boldsymbol{v}_{0} & =\boldsymbol{v}_{\mathrm{p}}-\boldsymbol{v}_{\text {orbit }} \\
& \approx\left(\Omega r_{\mathrm{s}}-v_{\text {orbit }}\right) \hat{\boldsymbol{e}}_{\text {orbit }},
\end{aligned}
$$

where $\Omega$ is the angular velocity of the central planet, $r_{s}$ the distance of the satellite from the planet's spin axis and $\hat{\boldsymbol{e}}_{\text {orbit }}$ the unit vector in orbital direction of the satellite (all three quantities as given in an inertial rest frame). In the moon-planet interaction both wings couple to the planet. In analogy with the exoplanetstar coupling, we will focus in the moon-planet interaction on the $c_{\mathrm{A}}^{-}$-wing as well.

We stress that in the analysis of this paper we use two separate frame of references, within which we apply two separate coordinate systems. One frame of reference is the rest frame of the obstacle which launches the Alfvén waves. In the rest frame of the obstacle the Alfvén wings are steady state under the assumption that the upstream conditions and the properties of the obstacle are steady state. The other frame of reference is rotating with the central body, i.e. the star or the central planet, at the radial distance of the exoplanet or the moon, respectively. In this frame of reference the Alfvén wings are time-dependent as an observer in this rest frame sees the wings being convected across the observer with time. These two frames of reference are connected by a transformation with a constant velocity $\boldsymbol{v}_{\mathrm{T}}$, whose direction and amplitude will be discussed in Sect. 2.1.3. We also use two separate coordinate systems applicable in each frame of reference (see Fig. 1). One coordinate system is called Alfvén wing system (Neubauer 1980). The Alfvén wing coordinate system in the reference frame of the obstacle is defined as follows: the $z$-axis is parallel to the Alfvén wing, i.e. parallel to $\boldsymbol{c}_{\mathrm{A}}^{-}$. The $y$-axis is along the $\boldsymbol{v}_{0} \times \boldsymbol{B}_{0}$ direction and the $x$-axis completes a right-handed coordinate system, i.e. lies in a plane defined by $\boldsymbol{v}_{0}$ and $\boldsymbol{B}_{0}$. The second coordinate system is called the $\boldsymbol{B}_{0}$ magnetic field system or primed system. In the rest frame of the obstacle it is defined as follows: the $z^{\prime}$ axis is anti-parallel to $\boldsymbol{B}_{0}$, the $y^{\prime}$ axis is in direction of $\boldsymbol{v}_{\text {orb }} \times \boldsymbol{B}_{0}$, and $x^{\prime}$ completes a right-handed coordinate system. The wing coordinate system (unprimed system) and the magnetic field system (primed system) within the same frame of reference are related through

$x=\cos \Theta_{\mathrm{A}} x^{\prime}-\sin \Theta_{\mathrm{A}} z^{\prime}$

$y=y^{\prime}$

$z=\sin \Theta_{\mathrm{A}} x^{\prime}+\cos \Theta_{\mathrm{A}} z^{\prime}$.
The angle $\Theta_{\mathrm{A}}$ describes the inclination of the Alfvén wing with respect to the background magnetic field (for the exact definition see (19)). The Alfvén wing coordinate system and the $\boldsymbol{B}_{0}$ magnetic field coordinate system in the rest frame of the rotating central body have coordinate axes in the same directions as the associated coordinate systems in the rest frame of the obstacle.

If $\left|\boldsymbol{v}_{\mathrm{T}}\right| \ll c$ with $c$ being the speed of light, the variables are related by a Galilei transformation between both frames of references. Denoting the variables in the rest frame of the obstacle with the superscript obst and variables in the rest frame of the rotating central body without any extra superscript, the plasma velocities in both frames are related by

$\boldsymbol{v}_{0}=\boldsymbol{v}_{0}^{\mathrm{obst}}-\boldsymbol{v}_{\mathrm{T}}$,

the electric fields by

$\boldsymbol{E}=\boldsymbol{E}^{\mathrm{obst}}+\boldsymbol{v}_{T} \times \boldsymbol{B}^{\mathrm{obst}}$,

and the magnetic fields by

$\boldsymbol{B}=\boldsymbol{B}^{\text {obst }}$

respectively.

\subsubsection{Model properties of the Alfvén wings}

If the planetary body including its atmosphere is electrically conductive and/or possesses a sufficiently strong internal magnetic field, the plasma flow in the vicinity of the body is slowed and the electric field is reduced. Simple models of the resultant electric field in sub-Alfvénic plasma interaction have been derived by Neubauer (1980, 1998), Saur et al. (1999), or Saur (2004), where the electric currents through the planetary bodies, ionospheres/atmospheres/magnetospheres are closed in the Alfvén waves, which are launched by the interaction.

In these models, the relative strength of the sub-Alfvénic interaction is characterized by a factor $\bar{\alpha}$. This factor assumes $\bar{\alpha}=0$ when no interaction takes place, i.e. the obstacles do not perturb the plasma flow, and $\bar{\alpha}=1$ for maximum interaction strength, i.e., the obstacles bring the exterior flow to a complete halt in its immediate vicinity. The factor is defined by

$$
\begin{aligned}
\bar{\alpha} & =1-E^{\mathrm{obst}} / E_{0}^{\mathrm{obst}} \\
& \approx 1-v^{\mathrm{obst}} / v_{0}^{\mathrm{obst}}
\end{aligned}
$$

i.e., it is related to how strongly the motional electric field and the plasma velocity in the vicinity of the obstacle is reduced due to the interaction.

In the model applied here the effects of the obstacles are simplified such that the resultant electric currents reduce the electric field $\boldsymbol{E}^{\text {obst }}$ within the obstacle of radius $R$ to a constant amplitude $(1-\bar{\alpha}) E_{0}^{\text {obst }}$. Writing the electric field in terms of the electric potential with $\boldsymbol{E}^{\text {obst }}=-\nabla \Phi^{\text {obst }}$ leads to

$$
\Phi^{i, \text { obst }}=(1-\bar{\alpha}) E_{0}^{\mathrm{obst}} y \quad r<R .
$$

Outside of the obstacle and perpendicular to $z$, the perturbation electric field decays as a two-dimensional dipole field

$$
\Phi^{e, \text { obst }}=E_{0}^{\text {obst }} y\left(1-\bar{\alpha} \frac{R^{2}}{r^{2}}\right) \quad r>R .
$$

This electric potential and the resultant electric field map into the Alfvén wings where they exhibit a two-dimensional structure with translational invariance along the wings similar to the 
other plasma properties in the wings (Neubauer 1980). In expressions (15) and (16), we use the radial distance $r$ of spherical coordinates with $x=r \cos \varphi$ and $y=r \sin \varphi$. The radius of the obstacle $R$ is in case of a nonmagnetized obstacle the radius of the planet including its atmosphere/ionosphere. In case of a magnetized body, the effective radius $R_{\text {eff }}$ depends on the properties of the internal and external magnetic field as detailed in Sect. 2.3.

In case of the obstacle being created by an ionosphere whose conductance is given by the Pedersen conductance $\Sigma_{\mathrm{P}}$ within $R$, then the interaction strength $\bar{\alpha}$ can be approximated (Neubauer 1998; Saur et al. 1999) by

$\bar{\alpha}=\frac{\Sigma_{\mathrm{P}}}{\Sigma_{\mathrm{P}}+2 \Sigma_{\mathrm{A}}}$.

The Pedersen conductance $\Sigma_{\mathrm{P}}$ is calculated by integrating the local Pedersen conductivity $\sigma_{\mathrm{p}}$ along the magnetic field lines through the planet's ionosphere beginning at the magnetic equator (e.g., Neubauer 1998; Saur et al. 1999). The Pedersen conductance is here assumed to be spatially constant within the ionosphere. In (17), we neglect the Hall conductance since the Hall currents are perpendicular to the electric field and thus only indirectly contribute to the energy budget of the interaction by modifying the overall electric and flow field. Note, also other physical reasons can slow and modify the flow near the obstacle. Examples are intrinsic magnetic fields (e.g., Ganymede see Kivelson et al. 1996a) or the effects of a plasma absorbing body such as Rhea, see Simon et al. 2012).

The Alfvén conductance $\Sigma_{\mathrm{A}}$ in (17) controls the maximum current which can be carried by an Alfvén wave. It is given after Neubauer (1980) by

$\Sigma_{\mathrm{A}}=\frac{1}{\mu_{0} v_{\mathrm{A}}\left(1+M_{\mathrm{A}}^{2}-2 M_{\mathrm{A}} \sin \Theta\right)^{1 / 2}}$.

The Alfvén wing is inclined with respect to the local background magnetic field $B_{0}^{\text {obst }}$ by the angle $\Theta_{\mathrm{A}}$ (see Fig. 1), which reads

$\sin \Theta_{\mathrm{A}}=\frac{\mu_{0} \Sigma_{\mathrm{A}} E_{0}^{\mathrm{obst}}}{B_{0}^{\mathrm{obst}}}=\frac{M_{\mathrm{A}} \cos \Theta}{\left(1+M_{\mathrm{A}}^{2}-2 M_{\mathrm{A}} \sin \Theta\right)^{1 / 2}}$.

The constancy of the Elsasser variables and a given electric field (e.g. with Eqs. (15) and (16)), constrains the magnetic field in an Alfvén wing after Neubauer (1980) to

$\boldsymbol{B}_{\perp}^{\text {obst }}=\left(\hat{z} \times \boldsymbol{E}^{\text {obst }}\right) \mu_{0} \Sigma_{\mathrm{A}}$

where $\perp$ denotes here the direction perpendicular to the wing, $\hat{z}$ is the unit vector in the $z$-direction and

$B_{z}^{\text {obst }}=-\sqrt{\left(B_{0}^{\text {obst }}\right)^{2}-\left(B_{\perp}^{\text {obst }}\right)^{2}}$.

\subsubsection{Model energy fluxes in Alfvén wing}

The Poynting flux, the kinetic and the thermal energy fluxes depend on the frame of reference. Even though the Alfvén wing is steady state in the rest frame of the obstacle for steady state upstream and planetary conditions, we are interested in the energy fluxes deposited into the central bodies. For the calculations of the energy fluxes, we therefore describe the plasma in a frame of reference moving with the central body (the star or the central planet) at a radial distance of the obstacle. We choose to calculate the fluxes at the radial distance of the obstacle because we are interested to determine the fluxes that are generated locally by the obstacle. With that choice there is no relative motion of the reference frame toward or away from the central body and the analysis plane through, which we calculate the energy fluxes does not move with respect to the central body. Note that a reference frame which would fully move with the plasma would generally not meet this criteria. The transformation from the rest frame of the obstacle to the rotating frame of the central body is given by

$$
\begin{aligned}
\boldsymbol{v}_{\mathrm{T}} & =\Omega r^{\text {obst }} \hat{\boldsymbol{e}}_{\text {orbit }}-\boldsymbol{v}_{\text {orbit }} \\
& =\left(\Omega r^{\text {obst }}-v_{\text {orbit }}\right) \hat{\boldsymbol{e}}_{\text {orbit }},
\end{aligned}
$$

where $\Omega$ is the angular velocity of the central body, $r^{\text {obst }}$ the distance between the central body and the obstacle, $\hat{\boldsymbol{e}}_{\text {orbit }}$ the unit vector in orbital direction of the obstacle (all three as seen from an inertial rest frame).

In the Alfvén wing coordinate system, the transformation $\boldsymbol{v}_{\mathrm{T}}$ from the rest frame of the obstacle into the rotating frame of reference is given by the velocity vector $\boldsymbol{v}_{\mathrm{T}}$ :

$$
\begin{aligned}
& v_{\mathrm{T}, x}=v_{0}^{\mathrm{obst}}\left(\cos \Theta \cos \Theta_{\mathrm{A}}+\gamma \sin \Theta_{\mathrm{A}}\right) \\
& v_{\mathrm{T}, y}=0 \\
& v_{\mathrm{T}, z}=v_{0}^{\mathrm{obst}}\left(\cos \Theta \sin \Theta_{\mathrm{A}}-\gamma \cos \Theta_{\mathrm{A}}\right)
\end{aligned}
$$

where $v_{0}^{\text {obst }}$ represents the relative velocity between the obstacle and the unperturbed plasma flow. This transformation is applicable for, both, the extra solar planets with relative plasma velocities given in (5) and the satellites at the outer planets with velocities given in (6). The factor $\gamma$ covers both cases and considers the different geometrical properties of the interaction at the moons or the extra solar planets. The factor $\gamma$ also describes the velocity of the plasma parallel to the background magnetic field.

For the extrasolar planets where the magnetic field is inclined by the Parker angle $\theta_{\mathrm{B}}$ with respect to the radial direction we find

$\gamma=-\tan \Theta_{\mathrm{B}} \cos \Theta$.

With this choice of $\gamma$ it can readily be shown that the resultant frame of reference is a frame that rotates with the angular velocity of the star $\Omega_{\star}$ at the radial distance of the extrasolar planet $r_{\text {exo }}$ assuming the Parker model with its frozen-in-field approximation for the stellar wind. The Parker angle $\Theta_{B}$, i.e. the angle between the radial direction and the direction of the magnetic field, is given by

$\tan \Theta_{\mathrm{B}}=\frac{\Omega_{\star} r_{\mathrm{exo}}}{v_{\mathrm{sw}}}$.

For the satellites in the outer solar system where the orbital velocities of the moons and the unperturbed magnetospheric fields are approximately perpendicular, we find

$\gamma=0$

and assume $\cos \Theta=0$. With this choice of transformation the new frame of reference is thus a frame which moves with the angular velocity of the central planet at the radial distance of the moon.

With the transformation of expressions (24) to (26), the resulting frame of reference is a frame where the relative velocity $\boldsymbol{v}_{0, \perp}$ perpendicular to the background magnetic field $\boldsymbol{B}_{0}$ vanishes, i.e. a frame of reference which "moves with the magnetic field". In the rotating frame of reference, the Alfvén wave travels parallel or anti-parallel to the unperturbed magnetic field and 


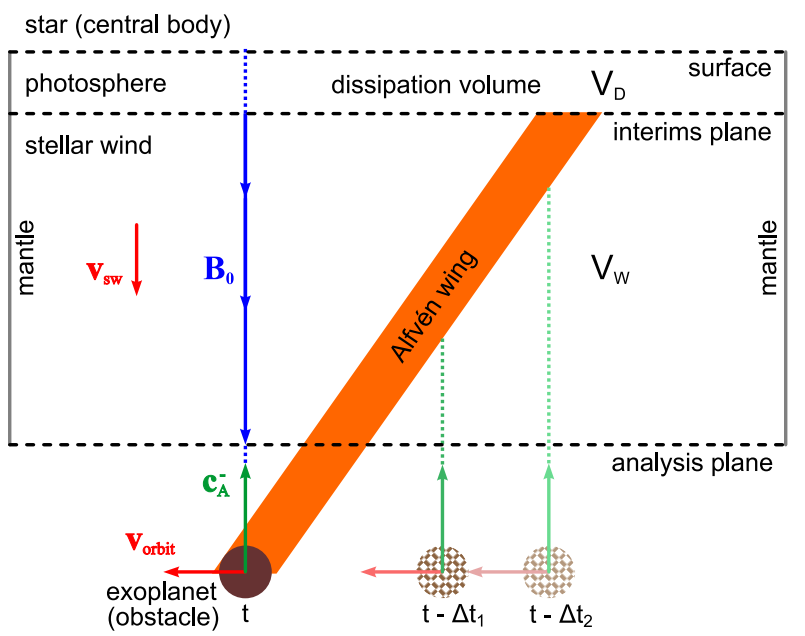

Fig. 2. Sketch of the Alfvén wing and its associated dissipation region for idealized stellar wind properties in a frame of reference rotating with the central body. The idealized orientations of the vector fields in the sketch resemble the situation when the radius of the star $R_{\star}$ is much larger than the effective radius of the extra solar planet $R_{\text {eff }}$ and the angular velocity of the star, the radial distance of the extra solar planet and the stellar wind velocity obey $\Omega_{\star} r_{\mathrm{exo}} / v_{\mathrm{sw}} \ll 1$. The sketch displays the wing at time $t$ and also indicates the time-variability of the wing in the rotating frame. At time $t$ the extra solar planet is situated at a location marked with a brown circle. The extra solar planet in this frame of reference was located at earlier times $t-\Delta t$ further to the right. The extra solar planet steadily launches Alfvén waves, which travel parallel to $\boldsymbol{B}_{0}$. The superposition of the wave packets launched at earlier times results in the snapshot of the wing at time $t$ displayed here.

thus the group velocity of the Alfvén wave is parallel or antiparallel to $\boldsymbol{B}_{0}$ (see Eq. (4)). The velocity component $\boldsymbol{v}_{0, \|}$ parallel to the background magnetic field is approximately zero in the case of the moon planet interaction. However, the velocity component $\boldsymbol{v}_{0, \|}$ parallel to the background magnetic field is non zero in the case of the extra solar planets and assumes a value of $\gamma v_{0}^{\text {obst }}$.

In the rotating frame of reference shifted by $\boldsymbol{v}_{\mathrm{T}}$ the unperturbed motional electric field $\boldsymbol{E}_{0}^{\text {obst }}$ vanishes and the net electric field $\boldsymbol{E}$ is given by expression (11). Note, however, that the velocity component $\gamma v_{0}^{\text {obst }}$ parallel to $\boldsymbol{B}_{0}$ makes a nonnegligible contribution in (11) because the cross product of $\boldsymbol{v}_{\mathrm{T}}$ is taken with the perturbed magnetic field $\boldsymbol{B}(\boldsymbol{x})$ and not with $\boldsymbol{B}_{0}$.

The time-constant, standing Alfvén wing in the obstacle frame of reference is a structure moving with $\boldsymbol{v}_{\mathrm{T}}$ and is thus timevariable in the rotating frame of reference. In the rotating frame, the group velocity of the Alfvén waves and the Elsasser variable $\boldsymbol{c}_{\mathrm{A}}^{-}$are parallel to the background magnetic field $\boldsymbol{B}_{0}$, but the Alfvén wing for a given time $t$ is still inclined by the angle $\Theta_{\mathrm{A}}$ with respect to $\boldsymbol{B}_{0}$ (see also Fig. 2).

In the rotating frame of reference and using the Alfvén wing coordinate system, we can now derive expressions for the Poynting flux $S$. Because the expressions are time-dependent in the rotating frame, we have to evaluate the Poynting flux at a certain time $t$. We can choose $t$ without loss of generality such that the center of the wing is located at $x^{\prime}=0$ and $y^{\prime}=0$ for a certain $z^{\prime}$. With (2), (11), (12), (15), (20), and (21) we find for the Poynting flux within the inner part of the Alfvén wing, i.e. for $r \leq R$

$S_{\mathrm{x}}^{\mathrm{i}} / S_{0}=-\Gamma_{1} \Gamma_{4}\left((-1+\bar{\alpha}) \cos \Theta_{\mathrm{A}}+\Gamma_{1}\right)$

$S_{\mathrm{y}}^{\mathrm{i}} / S_{0}=0$

$S_{\mathrm{z}}^{i} / S_{0}=(-1+\bar{\alpha}) \sin \Theta_{\mathrm{A}} \Gamma_{4}\left((-1+\bar{\alpha}) \cos \Theta_{\mathrm{A}}+\Gamma_{1}\right)$.
The Poynting flux in the inner part is spatially constant due to the model assumptions, which enter into expression (15). With (2), (11), (12), (16), (20), and (21), we find for the Poynting flux in the exterior part of the Alfvén wing, i.e. for $r>R$,

$$
\begin{aligned}
S_{\mathrm{x}}^{\mathrm{e}} / S_{0}= & -4 \bar{\alpha}^{2} R^{4} x^{2} y^{2} \sin ^{2} \Theta_{\mathrm{A}} \Gamma_{4} / r^{8} \\
& -\Gamma_{2}\left[-\Gamma_{3}+\Gamma_{3} \sin \Theta_{\mathrm{A}}\left(-\gamma \cos \Theta_{\mathrm{A}} / \cos \Theta+\sin \Theta_{\mathrm{A}}\right)\right. \\
& \left.+\Gamma_{4} \Gamma_{2}\right] \\
S_{\mathrm{y}}^{\mathrm{e}} / S_{0}= & -2 \bar{\alpha} R^{2} x y\left(\Gamma_{3} \sin ^{2} \Theta_{\mathrm{A}}+\Gamma_{2} \cos \Theta_{\mathrm{A}}\right) \Gamma_{4} / r^{4} \\
S_{\mathrm{z}}^{\mathrm{e}} / S_{0}= & \sin \Theta_{\mathrm{A}}\left\{4 \bar{\alpha}^{2} R^{4} x^{2} y^{2} \cos \Theta_{\mathrm{A}} \Gamma_{4} / r^{8}\right. \\
& -\Gamma_{3}\left[-\Gamma_{3}+\Gamma_{3} \sin \Theta_{\mathrm{A}}\left(-\gamma \cos \Theta_{\mathrm{A}} / \cos \Theta+\sin \Theta_{\mathrm{A}}\right)\right. \\
& \left.\left.+\Gamma_{4} \Gamma_{2}\right]\right\}
\end{aligned}
$$

with the abbreviations

$$
\begin{aligned}
& S_{0}=\frac{B_{0}^{2} v_{0} \cos \Theta}{\mu_{0}}, \\
& \Gamma_{1}\left(\bar{\alpha}, \Theta_{\mathrm{A}}\right)=\sqrt{1-(1-\bar{\alpha})^{2} \sin ^{2} \Theta_{\mathrm{A}}}, \\
& \Gamma_{2}\left(x, y, \bar{\alpha}, \Theta_{\mathrm{A}}\right)=\sqrt{1-\left(\bar{\alpha}^{2} R^{4}+2 \bar{\alpha} R^{2}\left(-x^{2}+y^{2}\right)+r^{4}\right) \frac{\sin ^{2} \Theta_{\mathrm{A}}}{r^{4}}}, \\
& \Gamma_{3}(x, y, \bar{\alpha})=1+\frac{\bar{\alpha} R^{2}\left(-x^{2}+y^{2}\right)}{r^{4}}, \\
& \Gamma_{4}\left(\Theta_{\mathrm{A}}, \Theta, \gamma\right)=\cos \Theta_{\mathrm{A}}+\gamma \sin \Theta_{\mathrm{A}} / \cos \Theta .
\end{aligned}
$$

In the Alfvén wings, also kinetic and thermal energy are convected away from the obstacle. Constancy of the Elsasser variable or Alfvén characteristic (see Eq. (4)) can be used to calculate the plasma velocity $\boldsymbol{v}=\boldsymbol{v}_{0}+\left(\boldsymbol{B}-\boldsymbol{B}_{0}\right) / \sqrt{\mu_{0} \rho}$ in the $\boldsymbol{c}_{\mathrm{A}}^{-}$ Alfvén wing. Using the same frame of reference and coordinate system as for the calculation of the Poynting flux, we find for the velocity field in the inner part of the wing (i.e., $r<R$ )

$v_{\mathrm{x}}^{\mathrm{i}} / v_{0}=\sin \Theta_{\mathrm{A}}\left(-\bar{\alpha} / M_{\mathrm{A}}-\gamma+\sin \Theta\right)$

$v_{\mathrm{y}}^{\mathrm{i}} / v_{0}=0$

$v_{\mathrm{z}}^{\mathrm{i}} / v_{0}=-\Gamma_{1} / M_{\mathrm{A}}+\cos \Theta_{\mathrm{A}}\left(1 / M_{\mathrm{A}}+\gamma-\sin \Theta\right)$

and in the exterior part (i.e. $r>R$ )

$$
\begin{aligned}
v_{\mathrm{x}}^{\mathrm{e}} / v_{0} & =\sin \Theta_{\mathrm{A}}\left(\sin \Theta-\gamma+\bar{\alpha} / M_{\mathrm{A}} R^{2}\left(-x^{2}+y^{2}\right) / r^{4}\right) \\
v_{\mathrm{y}}^{\mathrm{e}} / v_{0} & =-2 \bar{\alpha} / M_{\mathrm{A}} R^{2} x y \sin \Theta_{\mathrm{A}} / r^{4} \\
v_{\mathrm{z}}^{\mathrm{e}} / v_{0} & =\cos \Theta_{\mathrm{A}}\left(1 / M_{\mathrm{A}}+\gamma-\sin \Theta\right)-\Gamma_{2} / M_{\mathrm{A}} .
\end{aligned}
$$

This velocity field $\boldsymbol{v}$ determines the resultant kinetic energy flux $F_{\mathrm{k}}=1 / 2 \rho v^{2} \boldsymbol{v}$ and enthalpy flux $F_{\mathrm{T}}=5 / 2 k_{\mathrm{B}} 2 \rho / m T \boldsymbol{v}$, where $T$ is the plasma temperature, $k_{\mathrm{B}}$ the Boltzmann constant and $m$ the average ion mass of the flow. The factor of 2 enters if we assume that the ions and electrons have equal temperature. It provides additional contributions by the wing to the Joule dissipation in Eq. (1) in the rotating frame.

\subsubsection{Total energy fluxes toward the central body}

In this work we are interested in the energy sources $Q_{D}$ responsible for generating X-ray, UV, optical and IR emissions at the central body due to the sub-Alfvénic interaction with an obstacle. A likely candidate for this energy source is Joule dissipation of electromagnetic field energy feed by the Poynting flux. For further clarifications on how to determine the total Poynting flux 
communicated by the interaction toward the central body, it is helpful to return to Poynting's theorem (1). In MHD the electric field energy can be neglected compared to magnetic field energy as their ratio is proportional to $(v / c)^{2}$. The time derivates of the magnetic field energy also vanishes because $|\boldsymbol{B}|$ is constant within the Alfvén wing under the assumptions discussed in Sect. 2.1.2. We integrate (1) over a volume $V$ which starts "above" the obstacle but it includes the Alfvén wing and the part of the central body where the electromagnetic field energy is dissipated (see Fig. 2 for a simplified geometry). The resultant equation reads

$$
\int_{V} \mathrm{~d} V \nabla \cdot \boldsymbol{S}=-\int_{V} \mathrm{~d} V \boldsymbol{j} \cdot \boldsymbol{E}
$$

Within a volume $V_{W}$, which includes the Alfvén wing and which is outside of the obstacle and the central body, the term $\boldsymbol{j} \cdot \boldsymbol{E}$ describes the reversible work done by the electromagnetic field on the plasma and vice versa, i.e. the acceleration and deceleration of the flow. Within a volume $V_{\mathrm{D}}$ which includes the close proximity of the central body, we assume that $\boldsymbol{j} \cdot \boldsymbol{E}$ describes the irreversible, dissipative work done by the electromagnetic field. The Volume $V=V_{\mathrm{W}}+V_{\mathrm{D}}$ has a surface consisting of three parts: (1) a mantle; (2) an upper lit (or surface) which is located between the dissipation volume $V_{\mathrm{D}}$ and the interior of the central body and (3) a lower lit (or analysis plane) where the Alfvén wave generated by the interaction at the obstacle enters the volume. Applying Gauss Theorem, (47) reads

$$
\begin{aligned}
& \int_{\text {mantle }} \mathrm{d} \boldsymbol{A} \cdot \boldsymbol{S}+\int_{\text {upper lit }} \mathrm{d} \boldsymbol{A} \cdot \boldsymbol{S}+\int_{\text {lower lit }} \mathrm{d} \boldsymbol{A} \cdot \boldsymbol{S} \\
& =-\left.\int_{V_{\mathrm{W}}} \mathrm{d} V \boldsymbol{j} \cdot \boldsymbol{E}\right|_{\text {reversible }}-\left.\int_{V_{\mathrm{D}}} \mathrm{d} V \boldsymbol{j} \cdot \boldsymbol{E}\right|_{\text {dissipative }} .
\end{aligned}
$$

The surface integral of the Poynting flux through the mantle disappears when the mantle is displaced sufficiently far away from the wing because the Poynting flux in (33) to (35) decreases with $r^{-2}$ for large $r$ if $r$ characterizes the distance from the wing center to the mantle. Because the mantle area grows proportional to the product of $r H$, where $H$ is the length of the wing, the Poynting flux through the mantle decrease to zero with $r$ growing infinitely. The Poynting flux through the upper lit is zero because we assume that the flux is being absorbed in the dissipation volume $V_{\mathrm{D}}$. Thus only the Poynting flux through the lower lit is non zero. In this analysis we are not interested to measure the conversion of electromagnetic energy to mechanical energy, thus we need to choose the volume $V_{\mathrm{W}}$ such that the integral over $\left.\boldsymbol{j} \cdot \boldsymbol{E}\right|_{\text {reversible }}$ vanishes. Under this assumption (48) simplifies to

$$
\int_{\text {lower lit }} \mathrm{d} \boldsymbol{A} \cdot \boldsymbol{S}=-\left.\int_{V_{\mathrm{D}}} \mathrm{d} V \boldsymbol{j} \cdot \boldsymbol{E}\right|_{\text {dissipative }} \text {. }
$$

The integral on the right hand side of (49) represents the dissipated electromagnetic field energy and will be abbreviated by $Q_{\mathrm{D}}$. In (49) the surface integral on the left hand side describes the Poynting flux in direction of the outer normal to the lower lit of the volume $V_{\mathrm{W}}$. Because the obstacle generates an energy flux into the volume anti-parallel to the outer normal, we convert the sign in this integral and and call the ingoing flux through the lower lit: the Poynting flux through the analysis plane. Thus we can write

$$
Q_{\mathrm{D}}=\int_{\text {analysis plane }} \mathrm{d} \boldsymbol{A} \cdot \boldsymbol{S} \text {. }
$$

The orientation of the analysis plane needs to be chosen such that $\left.\int_{V_{\mathrm{W}}} \mathrm{d} V \boldsymbol{j} \cdot \boldsymbol{E}\right|_{\text {reversible }}=0$. The upper lit of the volume is assumed to be approximately parallel to the surface of the central body. We assume the magnetic field near the central body to be approximately perpendicular to the surface. In order for the volume integral over $\left.\boldsymbol{j} \cdot \boldsymbol{E}\right|_{\text {reversible }}$ to disappear the analysis plane also needs to be perpendicular to the magnetic field $\boldsymbol{B}_{0}$. This choice of the analysis plane is consistent with the group velocity of the Alfvén wave toward the central body being anti-parallel to $\boldsymbol{B}_{0}$ in the rotating rest frame, in which this analysis is performed. In the wing directed toward the central body, the wave travels parallel to

$-\hat{\boldsymbol{B}}_{0}=\hat{\boldsymbol{e}}_{z^{\prime}}=\left(-\sin \Theta_{\mathrm{A}}, 0, \cos \Theta_{\mathrm{A}}\right)$.

To calculate the fluxes through the analysis plane, we therefore multiply the Poynting fluxes and the respective velocity field components in Eqs. (30) to (35) and (41) to (46) with $\hat{\boldsymbol{e}}_{z^{\prime}}$.

The choice of the analysis plane perpendicular to the background magnetic field $\boldsymbol{B}_{0}$ as motivated in the previous paragraphs implies interesting consequences for the resulting Poynting flux. The Poynting flux through this analysis decreases with $r^{-4}$ for large distances from the wing center. The total, i.e. spatially integrated, Poynting flux therefore stays finite. The Poynting flux through any other analysis plane decreases with $r^{-2}$ for large distances from the wing center. The total Poynting flux in certain segments of these other planes therefore can assume infinite values.

To display the Poynting flux through the analysis plane, it is convenient to apply the primed coordinate system where $x^{\prime}$ and $y^{\prime}$ are perpendicular to $\boldsymbol{B}_{0}$. The primed Cartesian coordinates can also be expressed in spherical coordinates through $x^{\prime}=$ $r^{\prime} \cos \varphi^{\prime}$ and $y^{\prime}=r^{\prime} \sin \varphi^{\prime}$. The Alfvén wing cuts through the plane perpendicular to $\boldsymbol{B}_{0}$ inclined by the angle $\Theta_{\mathrm{A}}$ and thus a circle with radius $R$ turns into an ellipse given by $x^{\prime 2} \cos ^{2} \Theta_{\mathrm{A}}+$ $y^{\prime 2}=R^{2}$ or equivalently described by

$R_{\text {ellipse }}^{\prime 2}\left(\varphi^{\prime}\right)=\frac{R^{2}}{1-\sin ^{2} \Theta_{\mathrm{A}} \cos ^{2} \varphi^{\prime}}$

The total flux $S_{\text {total }}$ is given by the integral of the Poynting flux $S_{\|}^{\mathrm{i}}$ and $S_{\|}^{\mathrm{e}}$ over the plane perpendicular to $\boldsymbol{B}_{0}$

$$
S_{\text {total }}=\iint_{\left(x^{\prime} \cos \theta_{\mathrm{A}}\right)^{2}+y^{\prime 2} \leq R^{2}} S_{\|}^{\mathrm{i}} \mathrm{d} x^{\prime} \mathrm{d} y^{\prime}+\iint_{\left(x^{\prime} \cos \theta_{\mathrm{A}}\right)^{2}+y^{\prime 2}>R^{2}} S_{\|}^{\mathrm{e}}\left(x^{\prime}, y^{\prime}\right) \mathrm{d} x^{\prime} \mathrm{d} y^{\prime} .
$$

If the Alfvén Mach number $M_{\mathrm{A}}=v_{0} / v_{\mathrm{A}}$ assumes small values, i.e. in the limit $M_{\mathrm{A}} \rightarrow 0$, the total Poynting flux toward the star resulting from expression (53) can be strongly simplified to

$S_{\text {total }}=2 \pi R^{2} \bar{\alpha}^{2} \frac{E_{0} B_{0}}{\mu_{0}} M_{\mathrm{A}} \cos \Theta$,

which also can be rewritten as

$S_{\text {total }}=2 \pi R^{2} \frac{\left(\bar{\alpha} M_{\mathrm{A}} B_{0} \cos \Theta\right)^{2}}{\mu_{0}} v_{\mathrm{A}}$

i.e., the total flux is for small $M_{\mathrm{A}}$ proportional to the square of the magnetic field perturbation $\bar{\alpha} M_{\mathrm{A}} B_{0} \cos \Theta$ generated by the interaction times the Alfvén velocity $v_{\mathrm{A}}$ by which the energy is radiated away. As will be discussed in Sect. 2.2, the simplified expressions for the total Poynting flux in (54) or (55) might be useful approximations in a series of cases. These expressions differ from the expressions in Zarka (2007) and Lanza (2009) 


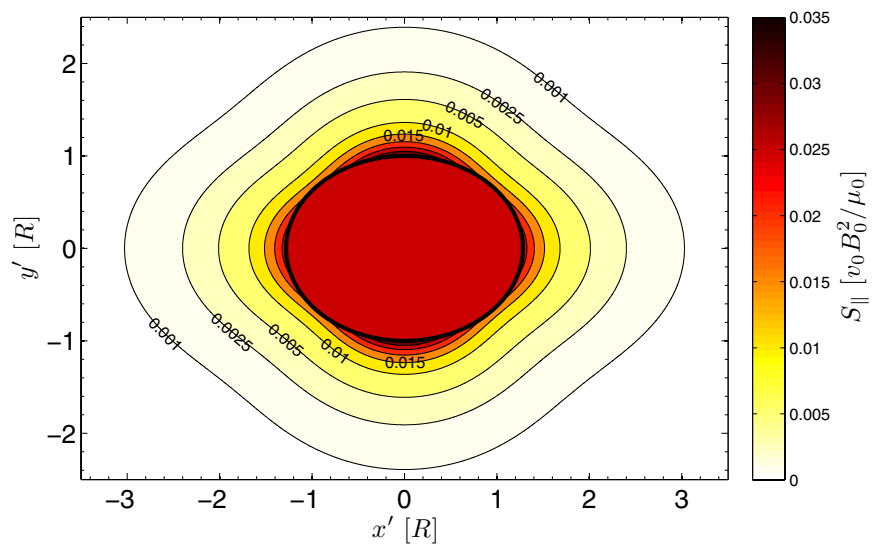

Fig. 3. Poynting flux through a plane perpendicular to $\boldsymbol{B}_{0}$ for Alfvén Mach number $M_{\mathrm{A}}=0.8$, interaction strength $\bar{\alpha}=0.2$, and flow direction perpendicular to $\boldsymbol{B}_{0}$, i.e. for $\Theta=0$.

next to other details by a factor of $2 v / v_{\mathrm{A}}$. We note that the energy dissipation in the central body and in the obstacle are not symmetric. The energy dissipation in the obstacles's ionosphere renders a maximum for interaction strength $\bar{\alpha}=1 / 2$ (Neubauer 1980), while the Poynting flux in expression (55) maximizes for maximum interaction strength $\bar{\alpha}=1$.

The kinetic and thermal energy fluxes are calculated similarly through a plane perpendicular to $\boldsymbol{B}_{0}$. In our rest frame, the background velocity $\boldsymbol{v}_{0}$, i.e. the velocity sufficiently far away from the obstacle is purely parallel to $\boldsymbol{B}_{0}$ and thus also antiparallel to the group velocity of the Alfvén wave. For extra solar planets $\boldsymbol{v}_{0}$ points away from the central star. We are interested in the total energy fluxes toward the central star and thus integrate the kinetic and thermal energy fluxes where the velocity vector parallel to $\boldsymbol{B}_{0}$ points toward the star, i.e. where $\boldsymbol{v}(\boldsymbol{x}) \cdot \hat{\boldsymbol{B}}_{0}<0$.

\subsection{Properties of the Poynting flux within the Alfvén wing}

We display the Poynting flux $S_{\|}=\boldsymbol{S} \cdot \hat{\boldsymbol{e}}_{z^{\prime}}$ in Fig. 3 for an Alfvén Mach number $M_{\mathrm{A}}=0.8$, an interaction strength $\bar{\alpha}=0.2$ and $\Theta=0$, i.e the magnetic field and plasma flow are perpendicular to each other. The Poynting flux is positive everywhere, i.e. points away from the obstacle toward the central body. In our model, the Poynting flux $S_{\|}^{\mathrm{i}}$ is constant in the inner part of the Alfvén wing, i.e. within the ellipse given by $r^{\prime}<R^{\prime}$ ellipse (see Eq. (52)). The Poynting flux achieves its maximum values on the flanks of the wing, i.e. near $x^{\prime}=0$ and $\left|y^{\prime}\right|>R$. The Poynting flux decreases as $r^{-4}$ at larger distance from the wing.

In Fig. 4 we show the total Poynting flux calculated with expression (53) as a function of the interaction strength $\bar{\alpha}$ for $M_{\mathrm{A}}=0.04, M_{\mathrm{A}}=0.4$ and $M_{\mathrm{A}}=0.66$ as solid lines. We also show for comparison the simple approximation of the Poynting flux given by the expressions in (54) or (55) as dashed lines. For small $M_{\mathrm{A}}$ the approximation fits the full expression very well, but even for large $M_{\mathrm{A}}$ both the full and simplified expression are still in reasonable agreement. Therefore the simple and "user friendly" expressions for the total Poynting flux provided in (54) or (55) might be used in a series of applications.

For large Alfvén Mach numbers $M_{\mathrm{A}}$, which obey the condition

$(1+\bar{\alpha})^{2} \sin ^{2} \Theta_{\mathrm{A}}>1$,

the argument of the root in (35) turns negative for selected locations of $x$ and $y$. In the case where the incident plasma

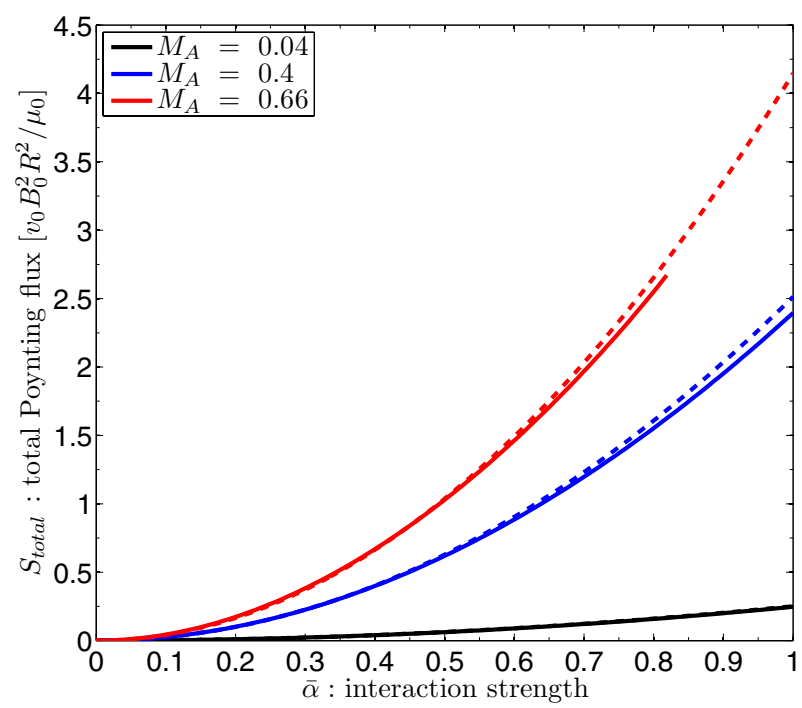

Fig. 4. Total Poynting flux through a plane perpendicular to $\boldsymbol{B}_{0}$ as a function of the interaction strength $\bar{\alpha}$ for different Alfvén Mach numbers $M_{\mathrm{A}}=0.04, M_{\mathrm{A}}=0.4$ and $M_{\mathrm{A}}=0.66$. The solid lines shows the solution of the full nonlinear expression (53) and the dashed lines the approximations given by (55). The background magnetic field $\boldsymbol{B}_{0}$ and the incident plasma flow $\boldsymbol{v}_{0}$ are assumed to be perpendicular, i.e. $\Theta=0$.

flow is perpendicular to the background magnetic field, i.e. $\Theta=0$, the condition simplifies to $M_{\mathrm{A}}>1 / \sqrt{\bar{\alpha}(2+\bar{\alpha})}$. Assuming additionally $\bar{\alpha}=1$, the condition reads $M_{\mathrm{A}}>1 / \sqrt{3}$ or $\Theta_{\mathrm{A}}>30^{\circ}$. For the parameter space where (56) is fulfilled, the expression in (35) cannot be used to calculate the Poynting flux. This effect is visible in Fig. 4 for the upper curves with $M_{\mathrm{A}}=0.66$. Under this circumstance the upper solid line only extends to $\bar{\alpha} \approx 0.8$. In case condition (56) applies in some of the sub-Alfvénic cases to be discussed in this work, a lower limit can be chosen by using a decreased interaction strength $\bar{\alpha}$ such that $(1+\bar{\alpha})^{2} \sin ^{2} \Theta_{\mathrm{A}}=1$.

The total Poynting flux also depends on the angle between the incident flow and the background magnetic field characterized by $\Theta$, which is defined such that $\Theta=0$, when $\boldsymbol{B}_{0}$ and $\boldsymbol{v}_{0}$ are perpendicular, see Fig. 1. In Fig. 5 we show the total Poynting flux as a function of the incident angle $\Theta$ for the Alfvén Mach numbers $M_{\mathrm{A}}=0.04, M_{\mathrm{A}}=0.4, M_{\mathrm{A}}=0.66$ and interaction strength $\bar{\alpha}=0.5$. The total Poynting flux is zero, when the flow is parallel or anti-parallel to the background magnetic field since the motional electric field in the rest frame of the plasma vanishes. In reality the obstacle will in this case still generate some perturbations, which are, however, very small compared to the case when $\boldsymbol{B}_{0}$ and $\boldsymbol{v}_{0}$ are approximately perpendicular. Within the planetary bodies under consideration in this work, $\Theta$ ranges from $0^{\circ}$ to $180^{\circ}$. However, the Poynting flux as a function of $\Theta$ is symmetric with respect to $\Theta=90^{\circ}$, i.e. $S_{\text {total }}\left(90^{\circ}+\Theta\right)=S_{\text {total }}\left(90^{\circ}-\Theta\right)$. In Fig. 5 we therefore display the range $0^{\circ} \leq \Theta \leq 90^{\circ}$ only, but add for completeness the range $-90^{\circ} \leq \Theta \leq 0^{\circ}$. The Alfvén wing angle $\Theta_{\mathrm{A}}$ is asymmetric with respect to the flow angle $\Theta=0$. The physical reason is that the plasma flow $\boldsymbol{v}_{0}$ has contribution parallel to $\boldsymbol{B}_{0}$ for $\Theta \neq 0$. If the flow is parallel to the Alfvén wave $\boldsymbol{v}_{\mathrm{A}}$ the tilt of the Alfvén wing is decreased, i.e. the wing is more aligned with $\boldsymbol{B}_{0}$. Figure 5 also shows that the approximated expression of (55) shown as dashed lines is for angles $\Theta \neq 0$ not under all circumstances an upper limit to the full expression (53) of the total Poynting flux shown as solid lines. 


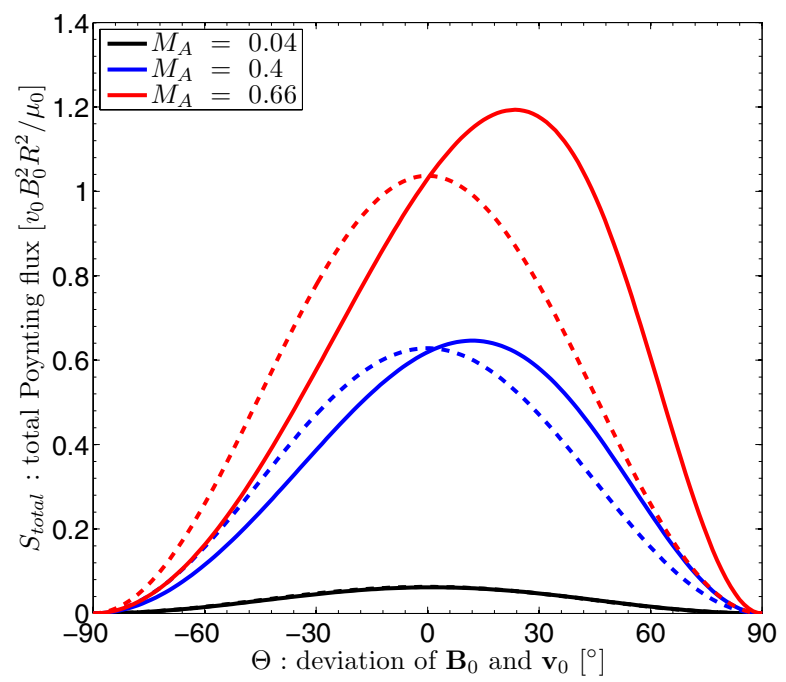

Fig. 5. Total Poynting flux as a function of the angle $\Theta$ between the background magnetic field $\boldsymbol{B}_{0}$ and the incident plasma velocity $\boldsymbol{v}_{0}$. The angle $\Theta$ is zero, when $\boldsymbol{B}_{0}$ and $\boldsymbol{v}_{0}$ are perpendicular (see Fig. 1). The Poynting flux is symmetric with respect to $90^{\circ}$. For the planetary bodies under consideration in this work, $\Theta$ falls into the range $0 \leq \Theta \leq 180^{\circ}$. Note that in this figure, we additionally show the range $-90^{\circ} \leq \Theta \leq 0^{\circ}$ for cases where such an orientation of the magnetic field and the velocity occur. The interaction strength and the Alfvén Mach number are chosen as $\bar{\alpha}=0.5$ and $M_{\mathrm{A}}=0.04, M_{\mathrm{A}}=0.4$ and $M_{\mathrm{A}}=0.66$, respectively. Solid lines are calculated with the full expression for the Poynting flux of Eq. (53) and dashed lines are calculated with the approximation of Eq. (55).

\subsection{Orientation of planetary magnetic field and effective radius of the obstacle}

In case the planetary body possesses an intrinsic magnetic field, the magnetic field environment near the planetary body and thus also the Poynting flux generated by the interaction with the surrounding flow is additionally modified. Here we assume that the internal magnetic field can be characterized by a dipole moment $\boldsymbol{M}_{\text {exo }}$ with equatorial field strength $B_{\text {exo }}$ on the surface of the planetary body. Next to the magnitude of the planetary magnetic field, its orientation with respect to the exterior field $\boldsymbol{B}_{0}$ plays a crucial role for the total Poynting flux. In Fig. 6 we show the magnetic field environment near the planet where we assume a planetary surface field $B_{\text {exo }}$ of 5000 nT with an associated dipole moment which is inclined by $45^{\circ}$ with respect to the stellar wind field $B_{0}$ of $100 \mathrm{nT}$. The magnetic field environment can be topologically divided into three areas. I: field lines which start on the planet and end on the planet, II: field lines which start on the planet and end on the star, and III: field lines which never intersect with the planet. The effective width of all field lines belonging to region I, when $\boldsymbol{B}_{0}$ and the magnetic dipole moment of the extrasolar planet $\boldsymbol{M}_{\text {exo }}$ are parallel is given by

$R_{\text {obst }}=R_{\text {exo }}\left(\frac{B_{\text {exo }}}{B_{0}}\right)^{1 / 3}=\left(\frac{\mu_{0}}{4 \pi} \frac{M_{\text {exo }}}{B_{0}}\right)^{1 / 3}$

where $R_{\text {exo }}$ is the radius of the extrasolar planet. This expression is often used to characterize the size of the planetary obstacle in the stellar wind (e.g. Lanza 2009). However, all field lines anchored in the planetary ionosphere from class II are slowed depending on the conductance of the planet's ionosphere, and thus establish the effective radius of the planet's magnetosphere $R_{\text {eff }}=1 / 2 D_{\text {eff }}$ as displayed in Fig. 6. The effective diameter $D_{\text {eff }}$ is the average distance at which field lines in regions

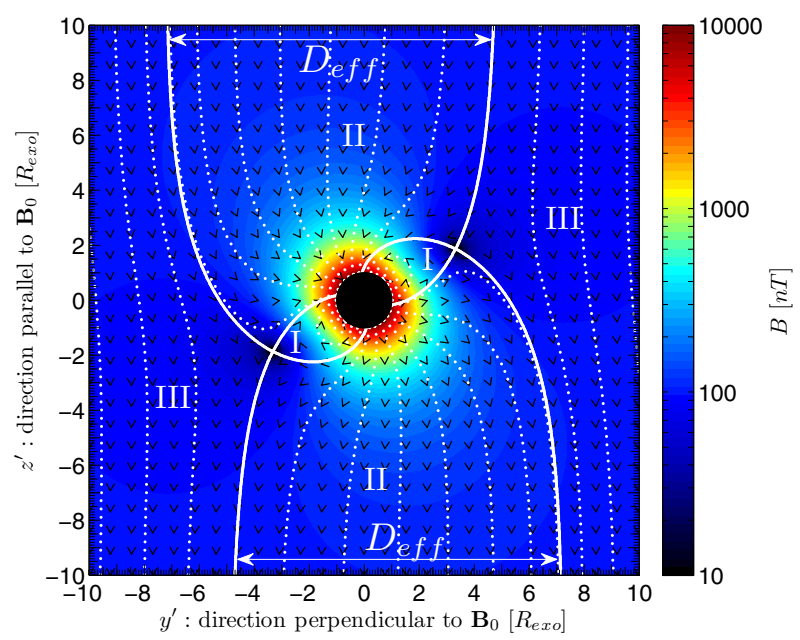

Fig. 6. Magnetic field topology and effective radius of Alfvén wings for oblique intrinsic and exterior field orientations. White dotted lines show magnetic field lines and black arrows indicate the magnetic field direction. The field strength is displayed color coded. The boundary, which separates closed field lines starting and ending on the planet from field lines connecting to the star, are displayed as solid white line. The distance of these field lines at larger distance from the planet establishes the effective diameter $D_{\text {eff }}$ of the Alfvén wings.

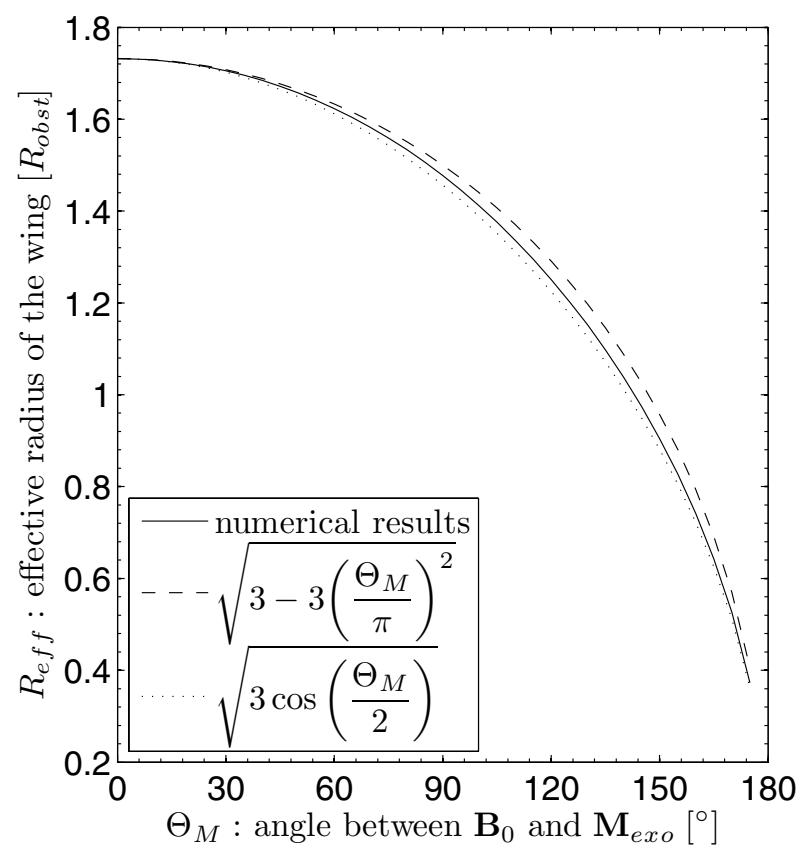

Fig. 7. Effective radius of Alfvén wing in units of $R_{\text {obst }}$ (see (57)) as a function of the orientation of the intrinsic to the external magnetic field (solid line). Two analytical approximations are shown as dashed and dotted lines.

II are separated at large distance from the planet. The effective radius as a function of the angle $\Theta_{M}$ between the planetary magnetic moment $\boldsymbol{M}_{\text {exo }}$ and the stellar wind magnetic field $\boldsymbol{B}_{0}$ is shown in Fig. 7. For arbitrary angle $\Theta_{M}$, the values of $R_{\text {eff }}$ need to be calculated numerically (solid line in Fig. 7). When the dipole moment and the stellar magnetic field are parallel, the effective radius is maximum and a factor of $\sqrt{3}$ larger than $R_{\text {obst }}$. Thus the effective area of the obstacle is a factor of 3 larger than the area determined by the closed field lines quantified by $R_{\text {obst }}$ in (57) and its assumptions. When the magnetic moment and the stellar 
Table 1. Total Poynting fluxes and properties at satellites for average magnetospheric values.

\begin{tabular}{|c|c|c|c|c|c|c|c|c|c|c|c|}
\hline Satellite & $\begin{array}{l}B_{0} \\
\text { nT }\end{array}$ & $\begin{array}{c}v_{0} \\
\mathrm{~km} \mathrm{~s}^{-1}\end{array}$ & $\begin{array}{c}n \\
\mathrm{~cm}^{-3}\end{array}$ & $\begin{array}{l}m_{\mathrm{ion}} \\
\mathrm{amu}\end{array}$ & $M_{\mathrm{A}}$ & $\begin{array}{l}R_{\text {eff }} \\
\mathrm{km}\end{array}$ & $\begin{array}{c}\Sigma_{\mathrm{P}, \mathrm{c}}^{\mathrm{a}} \\
\mathrm{S}\end{array}$ & $\bar{\alpha}$ & $\begin{array}{r}I_{\mathrm{obs}} \\
\mathrm{kR}\end{array}$ & $\begin{array}{c}S_{\text {obs }} \\
10^{9} \mathrm{~W}\end{array}$ & $\begin{array}{c}S_{\text {theo }} \\
10^{9} \mathrm{~W}\end{array}$ \\
\hline $\mathrm{Io}^{a, c}$ & 1790-2130 & 57 & $391-2330$ & 22 & $0.12-0.33$ & $1.3 \times 1822$ & 200 & $0.63-0.96$ & $5-700$ & $4-300$ & $288-1660$ \\
\hline Europa $^{a, c}$ & $387-473$ & 76 & $12-167$ & 18 & $0.11-0.49$ & $1.3 \times 1561$ & 30 & $0.06-0.85$ & $14-80$ & $0.1-8$ & $0.1-71$ \\
\hline Ganymede $^{a, c}$ & $69-116$ & 139 & $0.8-8$ & 14 & $0.19-0.98$ & $3.2-3.8 \times 2634$ & 16 & $0.09-0.79$ & $20-150$ & $0.1-15$ & $1-149$ \\
\hline Callisto $^{a}$ & $6-41$ & 192 & $0.001-0.5$ & 16 & $0.03-4.41$ & $1.3 \times 2410$ & 1000 & $0.02-1.0$ & - & - & $0.0001-5.2$ \\
\hline $\operatorname{Mimas}^{b}$ & 722 & 15.6 & 90 & - & 0.04 & $1.3 \times 195$ & $\sim 0$ & $\sim 0$ & - & - & $\sim 0$ \\
\hline Enceladus $^{b, d, e, f, g, h}$ & $h \quad 325$ & 26 & 70.5 & 17.6 & 0.13 & $1.3 \times 250$ & $\sim 10$ & $\sim 1$ & $0.45-1.55$ & $\sim 1-4 \times 10^{-3}$ & $\sim 0.2$ \\
\hline Dione $^{b, i}$ & 75 & 40 & 13 & 17 & 0.37 & $1.3 \times 560$ & 2 & $\sim 0.04$ & - & - & $\sim 0.0003$ \\
\hline Rhea $^{b, j}$ & 21 & 57 & 3.5 & 17 & 0.97 & $1.3 \times 765$ & 30 & $\sim 0.4$ & - & - & $\sim 0.02$ \\
\hline
\end{tabular}

Notes. Values either taken from or calculated based on ${ }^{(a)}$ Kivelson et al. (2004) ; ${ }^{(b)}$ Saur \& Strobel (2005); ${ }^{(c)}$ Wannawichian et al. (2010), Clarke et al. (2002), Gérard et al. (2006), Grodent et al. (2009), Bonfond et al. (2013), Prangé et al. (1996); ${ }^{(d)}$ Pryor et al. (2011); ${ }^{(e)}$ Saur et al. (2008); (f) Kriegel et al. (2009); ${ }^{(g)}$ Simon et al. (2011a); ${ }^{(h)}$ interaction strength $\bar{\alpha}$ was assumed to be maximum; ${ }^{(i)}$ Simon et al. (2011b); ${ }^{(j)}$ Santolík et al. (2011); ${ }^{(k)}$ Simon et al. (2009), Fluxes at Saturn's satellites are calculated with (55). $S_{\text {obs }}$ quotes the electron input energies derived from observation in the FUV. $S_{\text {theo }}$ quotes our calculated Poynting fluxes.

wind magnetic field are anti-parallel, i.e. the planetary field at the magnetic equator and the stellar wind magnetic field are parallel, the magnetosphere is closed. This effect has been noted by Ip et al. (2004) and Kopp et al. (2011), but not investigated quantitatively. For convenience we provide in Fig. 7 two expressions which approximate the numerical values. Thus the relative orientation of the planetary magnetic moment and the stellar wind magnetic field has strong effects on the magnitude of the resulting Poynting flux launched at the planet.

\section{Poynting fluxes generated by the satellites of Jupiter and Saturn}

In our solar system, sub-Alfvénic plasma interactions are observed at a number of satellites in the outer solar system (see Sect. 1). We therefore can use these observational constraints to benchmark our derived expressions of the previous section and predict luminosities of possible satellite footprints which have not yet been observed.

\subsection{Jupiter's Galilean satellites}

At the Galilean satellites of Jupiter, the Poynting fluxes generated by the interactions are time-dependent and depend on the satellites' positions in Jupiter's magnetosphere because Jupiter's magnetic moment is inclined by $\sim 10^{\circ}$ with respect to its spin axis (e.g. Kivelson et al. 2004). Therefore magnetic field strengths, plasma densities and interaction strengths vary with the satellites' positions in Jupiter's rotating magnetosphere conveniently measured in system III longitude.

While most quantities in the expressions for the Poynting fluxes (30) and (35) have been measured by spacecraft and are listed in Table 1, the relative strength of the plasma interaction $\bar{\alpha}$ is not a directly observable quantity, but can be estimated with the Pedersen conductance of the satellites' ionospheres $\Sigma_{\mathrm{P}}$ and the Alfvén conductance $\Sigma_{\mathrm{A}}$ through (17). The latter two quantities are not directly measurable either, but the Alfvén conductance $\Sigma_{\mathrm{A}}$ is given by (18) and depends dominantly on the observationally constrained plasma densities and magnetic field strengths. The Pedersen conductance $\Sigma_{\mathrm{P}}$ is the local Pedersen conductivity integrated along the magnetic field lines through the satellites' ionospheres. The Pedersen conductances have been modeled in various studies (e.g. Saur et al. 1999, 2002; Kivelson et al. 2004). For Jupiter's satellites we use the maximum, spatially averaged ionospheric conductances $\Sigma_{\mathrm{P}, \mathrm{c}}$ listed in Kivelson et al. (2004) as the values in the center of Jupiter's plasma sheet. Because the Pedersen conductivity is proportional to the plasma density in the ionosphere, we scale the variation of the Pedersen conductance $\Sigma_{\mathrm{P}}$ relative to its values in the center of the Jovian plasma sheet $n_{\mathrm{c}}$ with

$\Sigma_{\mathrm{P}} \approx\left(\frac{n}{n_{\mathrm{c}}}\right)^{\kappa} \Sigma_{\mathrm{P}, \mathrm{c}}$.

When the torus plasma enters the satellites' ionospheres unmodified, $\kappa=1$. An enhanced plasma density through enhanced electron impact ionization can be considered by $\kappa>1$.

Due to uncertainties in the detailed spatial variations of the plasma density in Jupiter's magnetosphere at the locations of the Galilean satellites as a function of system III longitude, we apply in the following Sects. 3.1.1 to 3.1.4 two different models of the plasma densities for each satellite. One set of models is individually constructed based on various sources in the literature and the other model is after Bagenal \& Delamere (2011), who provide a model for the plasma properties of Jupiter's magnetosphere. Due to uncertainties in the variability of the interaction strength as a function of system III longitude, we also investigate its effect on the energy fluxes. Therefore we use the density model of Bagenal \& Delamere (2011) and vary the interaction strength by choosing $\kappa=1$ and $\kappa=2$ in (58). For each of the Galilean satellites we therefore apply three models, respectively, to study the variability of the footprint brightness and their dependences on the plasma properties in the vicinity of the Galilean satellites.

\subsubsection{Poynting fluxes: lo}

The resultant Poynting fluxes at Io as a function of system III are displayed in Fig. 8 in the first panel. The Poynting flux calculated with the density model extracted in Jacobsen (2011) based on Bagenal (1994) is shown as dashed solid line with $\kappa=2$. Additionally, we use the density model by Bagenal \& Delamere (2011) with $\kappa=1$ and $\kappa=2$ and display the resultant Poynting fluxes with the thin blue and the thick black line, respectively. The magnetic field model is based on the composite model including Jupiter's internal field and the plasma sheet contributions assembled in Seufert et al. (2011). For the interaction strength $\bar{\alpha}$ we use (17) and (58) with $\Sigma_{\mathrm{P}, \mathrm{c}}=200 \mathrm{~S}$ in the center of the plasma sheet (Saur et al. 1999; Kivelson et al. 2004). We assume an effective radius due to Io's ionosphere of $R_{\mathrm{eff}}=1.3 R_{\mathrm{Io}}$, an 


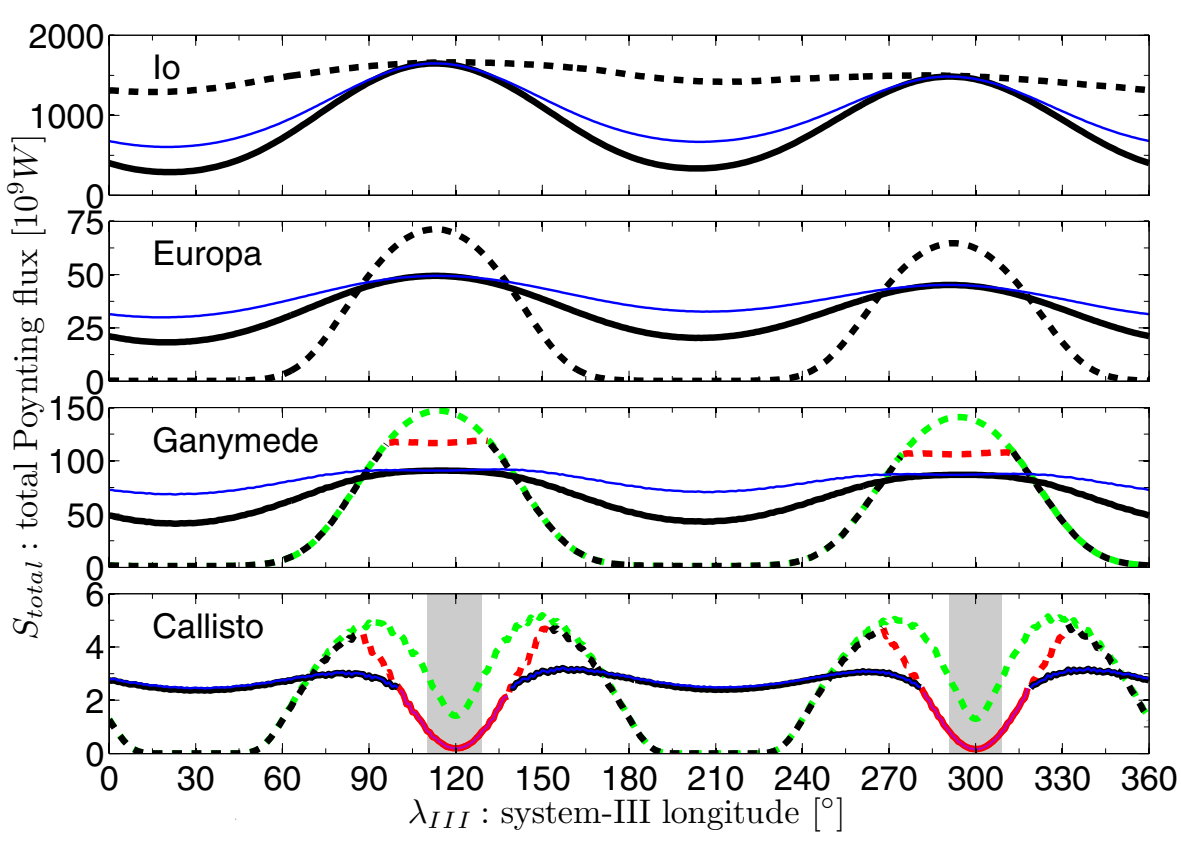

Fig. 8. Total Poynting flux generated by the Galilean satellites as a function of system III longitude for different models of the plasma density and interaction strength. Solid lines display the Poynting flux with magnetospheric density models after Bagenal \& Delamere (2011) calculated with two different assumptions of the interaction strength $\bar{\alpha}$ controled by $\kappa=1$ (thin blue) and $\kappa=2$ (thick black). The dashed line displays the Poynting flux for individual magnetospheric plasma models, where the dashed black lines are calculated with the full expression given in (53), while the dashed green and the dashed red lines represent upper and lower limits when condition (56) is met. The grey area shows cases where the interaction is expected to be super-Alfvénic, i.e. $M_{\mathrm{A}}>1$. More details see explanations in the main text. Results are discussed for each satellite individually in Sects. 3.1.1 to 3.1.4. average mass for the torus plasma of $22 \mathrm{amu}$, and $\Theta \approx 0$ (Saur et al. 1999; Kivelson et al. 2004).

Average values for the Poynting flux are on the order of $\sim 1 \times$ $10^{12} \mathrm{~W}$. These values can be compared with Hubble Space Telescope (HST) observations. The observed intensities of Io's footprint in the far-ultraviolet (FUV, i.e. $120-180 \mathrm{~nm}$ ) typically lie on the order of several $100 \mathrm{kR}$, where $1 \mathrm{kR}=10^{3}$ Rayleigh correspond to $10^{13}$ photons $\mathrm{m}^{-2} \mathrm{~s}^{-1}$ into $4 \pi$ steradians (Clarke et al. 2002). An emission output in the FUV of $1 \mathrm{kR}$ requires an input energy flux by energetic electrons of $\sim 10^{-4} \mathrm{~W} \mathrm{~m}^{-2}$ (e.g., Gérard et al. 2006). In the FUV, an emission of $1 \mathrm{kR}$ corresponds to an energy flux of $\sim 10^{-5} \mathrm{~W} \mathrm{~m}^{-2}$. This implies a conversion/efficiency factor of $\sim 10 \%$ of the energy input flux by energetic electrons compared to the energy output as FUV photons. If the extreme-ultraviolet spectrum (EUV, i.e. 80-120 nm) is included in addition to the FUV, the conversion factor is $\sim 20 \%$ (Gustin et al. 2012; Bonfond et al. 2013). Io's main footprint displays a brightness in the FUV in the range of 5-700 kR (e.g., Clarke et al. 1996, 1998; Gérard et al. 2006; Wannawichian et al. 2010). The required electron input power to generate these footprint fluxes was derived to lie in the range $4-300 \times 10^{9} \mathrm{~W}$ (e.g., Prangé et al. 1996; Clarke et al. 1996; Gérard et al. 2006). Very recently, Bonfond et al. (2013) reanalyzed a large set of HST observations of Io's footprint and found a maximum vertical brightness of approximately $\sim 2-20 \times 10^{3} \mathrm{kR}$ in the EUV+FUV with an associated energy input in the range of $\sim 25-200 \times 10^{9} \mathrm{~W}$. Our modeled Poynting fluxes lie in the range of $288-1660 \times 10^{9} \mathrm{~W}$ (see Table 1). Comparison of the observationally derived energy input fluxes with our Poynting fluxes imply that on the order of $\sim 10 \%$ of the total Poynting flux of Io's Alfvén wave/wing is converted into accelerated electrons to generate Io's main footprint. The rest of the wave energy is (a) partially reflected on its way to Jupiter (Chust et al. 2005; Hess et al. 2010a, 2013), is (b) partially distributed over a larger area including multiple spots and the downstream auroral trail, and is (c) partially converted into other forms of plasma energy, such as heating. But succinctly, the overall energy in the Poynting flux originating at Io is sufficient to generate Io's auroral footprint.

The quoted brightness of Io's UV footprints show significant variability. Next to variations of an apparent random nature, a systematic trend in the total UV intensity is due to Io's position with respect to the Jovian plasma sheet with typical values varying by a factor of $\sim 5$ from $\sim 50 \mathrm{kR}$ to $\sim 250 \mathrm{kR}$ according to Wannawichian et al. (2010). Depending on the input torus model used in our calculations, we see a very weak variability of $\sim 20 \%$ (dashed line in upper panel of Fig. 8) up to a variability by a factor of $\sim 5$ as a function of system III longitude (solid lines in upper panel of Fig. 8). The strongest contribution to the variability in the locally generated Poynting fluxes is due to the variable density with weaker contributions due to the variable interaction strength $\bar{\alpha}$, whereas the magnetic field strength at Io varies only modestly as a function of system III. Additional effects of the far-field likely contribute to a stronger variability. According to Jacobsen et al. (2007), the stronger the interaction the more nonlinear is the reflection of Io's Alfvén waves at the electron acceleration region or Jupiter's ionosphere characterized by its Pedersen and Hall conductances. For a fully nonlinear interaction, the Alfvén waves are reflected back to Io within the original Alfvén waves. In this case no multiple footprints downstream are seen, but only a downstream tail, as the wave is reflected within itself (Gérard et al. 2006; Jacobsen et al. 2007). Therefore the wave energy of the multiple spots is combined, when Io is in the center of the current sheet. Bonfond et al. (2008) report the discovery of a leading spot, i.e. a precursor spot, when Io is outside the center of the torus. When Io is in the center of the torus, the leading spot will overlap with Io's main spot and thus will enhance its brightness as well. A fraction of the wave energy at Io will be partly reflected and filamented while traveling along the inhomogeneous plasma densities and magnetic fields (Wright \& Schwartz 1989; Chust et al. 2005; Jacobsen et al. 2007; Hess et al. 2010a, 2011a). The strength of the wave reflection, transmission and filamentation also depends on Io's position in the torus and will contribute to the variably of the brightness (Hess et al. 2013).

\subsubsection{Poynting fluxes: Europa}

Clarke et al. (2002) report the first observation of an auroral footprint of Europa with brightnesses of a few $10 \mathrm{kR}$ in the FUV. Grodent et al. (2006) measure $14 \mathrm{kR}$ for the main spot, but also 
observe an auroral tail. Wannawichian et al. (2010) see variations in the FUV spot brightness between 20 and $80 \mathrm{kR}$. The total emitted power derived by Grodent et al. (2006) is $8 \times 10^{8} \mathrm{~W}$ with a spot size corresponding to an upper limit of 15 times the diameter of Europa. Clarke et al. (2002) report energy input fluxes for Europa's footprint of $1-5 \times 10^{8} \mathrm{~W}$.

We calculate the total Poynting flux as function of $\lambda_{\text {III }}$ with the following parameters for three different models: we use $\Sigma_{\mathrm{P}, \mathrm{c}}=30 \mathrm{~S}$ which corresponds to $\bar{\alpha}_{\mathrm{c}} \sim 0.8$ in the center of the plasma sheet (Kivelson et al. 2004). We assume an extension of Europa's interaction region of $R_{\mathrm{eff}}=1.3 R_{\text {Europa }}$ (Saur et al. 1998). The magnetic field is calculated similar to Io with the model by Seufert et al. (2011). The dashed curve in Fig. 8 uses torus electron number densities from Schilling (2006) with an average ion charge of 1.5 elementary charges and average mass of 18 amu (Kivelson et al. 2004). We extract the ion density assembled in Schilling (2006) by an analytic expression of the form $n_{0}+n_{1} \sin ^{\gamma}\left(\lambda_{\mathrm{III}}-\lambda_{0}\right)$, with the free parameters $n_{0}, n_{1}, \lambda_{0}$ and $\gamma$. For Europa we use for the electron density $n_{0}=18 \mathrm{~cm}^{-3}$, $n_{1}=232 \mathrm{~cm}^{-3}, \lambda_{0}=22.5^{\circ}$, and $\gamma=4$ based on the values in Schilling (2006) and we use $\kappa=2$. The solid blue and black lines use the density model of Bagenal \& Delamere (2011) with $\kappa=1$ and $\kappa=2$, respectively.

The resulting total Poynting fluxes as function of system III are shown in the second panel of Fig. 8. The total Poynting flux clearly varies as a function of $\lambda_{\text {III }}$ with the maximum values when Europa is in the center of the Jovian plasma sheet. Our model results are consistent with the derived energy input fluxes by HST of $0.1-8 \times 10^{9} \mathrm{~W}$ (Clarke et al. 2002; Grodent et al. 2006). We find model fluxes in the range of $\sim 0.1-71 \times 10^{9} \mathrm{~W}$. These observed values are on the order of $\sim 10 \%$ of the total Poynting flux generated at Europa, i.e. similar to Io only a fraction of the total Poynting flux is converted into electron input energies to generate the UV emissions of the central auroral spot. The brightness variability in our model is different for each model. The strongest impact on the variability is due to the variability of the plasma density as a function of system III. The variability of the local interaction strength also contributes to the variability of the Poynting flux and the nonlinearity of the farfield interaction similar to the discussion at Io might play a role as well.

\subsubsection{Poynting fluxes: Ganymede}

Clarke et al. (2002) first observed the Ganymede footprints in Jupiter's ionosphere/atmosphere with a brightness of a few $10 \mathrm{kR}$ which requires an energy input of $0.1-0.5 \times 10^{9} \mathrm{~W}$. Grodent et al. (2009) derive values for the energy input in the range of $2-15 \times 10^{9} \mathrm{~W}$ where the auroral footprint matches an area with a diameter of $8-20 R_{\text {Ganymede }}$. Wannawichian et al. (2010) see brightness variations between $\sim 50-150 \mathrm{kR}$.

We calculate the total Poynting flux as function of $\lambda_{\text {III }}$ with the following model parameters: we use $\Sigma_{\mathrm{P}, \mathrm{c}}=16 \mathrm{~S}$ which is 4 times higher compared to the value referenced in Kivelson et al. (2004), but it leads to more realistic values of the polar flow velocity in agreement with Jia et al. (2009). We assume $\kappa=2$. For the torus ion density, we use for the model displayed as dashed line in Fig. 8 a similar dependency as for Europa with $\gamma=4, n_{0}=1 \mathrm{~cm}^{-3}, n_{1}=7 \mathrm{~cm}^{-3}$, and $14 \mathrm{amu}$ as average mass (Kivelson et al. 2004). This model can be regarded as an upper limit for the variability as function of system III. The solid blue and the black lines are calculated with density models from Bagenal \& Delamere (2011) and $\kappa=1$ and $\kappa=2$, respectively. The magnetic field model is after Seufert et al. (2011). Ganymede possesses an intrinsic magnetic moment and thus a mini-magnetosphere within Jupiter's large magnetosphere (Kivelson et al. 1996a; Neubauer 1998). The intrinsic magnetic field significantly enlarges the size of the obstacle to the flow. As discussed in Sect. 2.3 the size of the open-closed field line area given by (57) is in case when the magnetic moment and the external magnetic field are parallel enhanced by a factor of $\sqrt{3}$. Thus the effective radius of Ganymede as a source of Alfvén waves is $R_{\mathrm{eff}}=\sqrt{3}\left(B_{p} / B_{\mathrm{sw}}\right)^{1 / 3} R_{\text {Ganymede }}$, which assumes values in the range of $\approx 3.2-3.8 \times R_{\text {Ganymede }}$, depending on Ganymede's position with respect to the magnetospheric current sheet. Thus the effective area and the resulting Poynting flux are enhanced by roughly a factor of 10 compared to $\pi R_{\text {Ganymede }}^{2}$ due to Ganymede's internal magnetic field.

The total Poynting flux as a function of system III is shown in the third panel of Fig. 8 for our three different models. The total Poynting flux assumes values as high as $\sim 1.5 \times 10^{11} \mathrm{~W}$ with the maximum values at $\lambda_{\text {III }}$ where Ganymede is in the center of the Jovian plasma sheet. In the center of the plasma sheet our solution is in the range where it does not obey the condition (56) any more. In this case we use the approximate expression for the Poynting flux in (55) as an upper limit (dashed green curve), and we lower $\sin \left(\Theta_{\mathrm{A}}\right)$ in (53) (to avoid altering $\bar{\alpha}$ ) until condition (56) is met as a lower limit (dashed red curve).

The observationally derived energy input fluxes in the range of $0.1-15 \times 10^{9} \mathrm{~W}$ (Clarke et al. 2002; Grodent et al. 2009) are consistent with our model fluxes which lie in the range of $1-149 \times 10^{9} \mathrm{~W}$. Thus also at Ganymede, our predicted Poynting flux is sufficiently large to account for the auroral footprint energy budget. Similarly to Io on the order of $\sim 10 \%$ of the generated Poynting flux is converted into electron acceleration to generate Europa's footprint. Our predicted variability depends strongly on the density model and the model of the interaction strength. Our model variabilities are within the observed variability ranges by Clarke et al. (2002); Grodent et al. (2006); Wannawichian et al. (2010). Also here similar to Io, wave reflection and the nonlinear feedback of the reflected waves might additionally contribute to the variability.

\subsubsection{Poynting flux: Callisto}

Callisto's footprints have not yet been fully confirmed observationally. They likely lie within Jupiter's main auroral oval which has a typical brightness of a few $100 \mathrm{kR}$. First observational hints for the existence of Callisto's footprints where presented by Clarke et al. (2011). Here we provide predictions for footprint brightness and variability, as Jupiter's main auroral oval can shift its location due to variable internal sources and varying solar wind conditions and thus might very occasionally not overlap with a possible footprint of Callisto.

We calculate the total Poynting flux as a function of $\lambda_{\text {III }}$ for three different models. The dashed black line is calculated with a similar density dependence as for Europa and Ganymede with minimum and maximum values ranging between 0.001 and $0.5 \mathrm{~cm}^{-3}$ and an average mass of $16 \mathrm{amu}$ (Kivelson et al. 2004) and apply $\kappa=2$ for this model. The solid blue and the black lines are calculated with density models after Bagenal \& Delamere (2011) and $\kappa=1$ and $\kappa=2$, respectively. We use in the center of the plasma sheet $\Sigma_{\mathrm{P}, \mathrm{c}}=1000 \mathrm{~S}$ (Kivelson et al. 2004), which corresponds to an interaction strength of $\bar{\alpha}_{\mathrm{c}}>0.99$ (Strobel et al. 2002). The magnetic field model is after Seufert et al. (2011). The resulting Poynting fluxes are displayed in the 
fourth panel of Fig. 8. The total Poynting flux assumes values up to $\sim 5 \times 10^{9} \mathrm{~W}$. Near the center of the plasma sheet (56) is violated. Thus we calculate similar to the procedure at Ganymede an upper limit (green dashed curve) and a lower limit (red curve). The grey area indicates cases where the interaction is superAlfvénic, i.e. $M_{\mathrm{A}}>1$. The theoretical model of the Alfvén wings by Neubauer (1980) which we apply has, however, been developed for $M_{\mathrm{A}}<1$. All three models predict that Callisto's Poynting fluxes are not maximum in the center of the current sheet. The reason is that at radial distances of Callisto the magnetic field strength in the center of the current sheet is strongly reduced and even compensates the effects of the enhanced plasma density within the current sheet.

\subsubsection{Comparison and comments}

Zarka (2007) and Hess et al. (2011a) estimate values for the energies in the interaction of the satellites with Jupiter's magnetosphere. Our derived values for the Poynting flux are on the same order but somewhat smaller than those in Zarka (2007) since the expressions in Zarka (2007) consider the energy flux into the satellites' ionospheres or the energies dissipated within the satellites' ionospheres, which are generally larger than the energy radiated away from the satellites, which we calculate here. Hess et al. (2010b, 2013) also investigate, based on simplified expressions for the local interaction, the variability of the luminosity of the satellites' footprints focusing on the far-field, i.e. the reflection and filamentation of the waves.

An additional important component which creates variability of the footprint brightness as a function of system III, might be the plasma properties in the region where magnetic field energy is converted into particle acceleration. For Jupiter the auroral acceleration region is thought to occur a few Jovian radii above Jupiter's ionosphere. In this region the density of ions and energetic electrons might vary with implications on the variability of the footprint brightness as discussed for example in Bonfond et al. (2007); Hess et al. (2011b).

Note that the observationally derived brightnesses and estimated input fluxes for the satellites footprints by the various authors where partially derived under somewhat different assumption and with somewhat different analysis techniques.

Succinctly, the overall energy flux values in the Poynting fluxes originating at the Galilean satellites are sufficient to generate their auroral footprints.

\subsection{Poynting flux: Saturn's inner icy satellites}

At Saturn so far only an Enceladus footprint has been observed by Pryor et al. (2011). The observed spot brightness in the UV ranges from 0.45 to $1.55 \mathrm{kR}$ with the size of the spots possibly extending $20 R_{\text {Enceladus }}$ downstream. With the values of Table 1, we predict a Poynting flux of $\sim 200 \times 10^{6} \mathrm{~W}$. For this value we assumed a maximum interaction strength and a fairly large size of the obstacle. The value thus rather needs to be considered an upper limit. Based on the observed brightness a footprint size corresponding to $1.3 R_{\text {Enceladus }}$ with a downstream extension of $20 R_{\text {Enceladus }}$ would lead to an auroral electron energy input of $\sim 1-4 \times 10^{6} \mathrm{~W}$ assuming $1 \mathrm{kR}$ requires an energy input of $1 \times 10^{-4} \mathrm{~W} \mathrm{~m}^{-2}$ (Pryor et al. 2011). Thus the predicted Poynting flux is larger and sufficient to account for the UV observations and its large extension. We note that at Enceladus the neutral gas environment possesses a north south asymmetry due the location of the water plume near Enceladus' south pole (Dougherty et al. 2006; Tokar et al. 2006). This asymmetry in the gas generates asymmetric Alfvén wings and associated asymmetric Poynting fluxes (Saur et al. 2007). Using the asymmetric expressions for the electric fields derived in Saur et al. (2007) or Simon et al. (2011a), these asymmetric fluxes can be calculated in the same manner as in Sect. 2. Due to the electromagnetic coupling of Enceladus' southern and northern hemispheres (Saur et al. 2007), we expect even in the case where the south polar plume would be located only near and "just below" Enceladus' south pole, still Poynting flux being radiated into the northern hemisphere of Saturn's magnetosphere.

Based on the values in Table 1, among the inner icy satellites of Saturn, only Rhea and Dione might produce a Poynting flux which could possibly lead to observable footprints in case of sufficiently long exposure times. Both, Rhea and Dione have sputtering generated atmospheres/exospheres (Saur \& Strobel 2005; Teolis et al. 2010; Simon et al. 2011b) which is a root cause for the generation of Alfvén waves. The Poynting fluxes at the satellites of Saturn might vary as a function of time due to varying magnetospheric properties and/or varying neutral atmosphere densities caused by a time variable radiation belt (Roussos et al. 2008; Simon et al. 2011b). Note that Simon et al. (2012) show that the dominant part of the magnetic field perturbations observed by the Cassini spacecraft near Rhea is due to plasma absorption effect caused by the solid body of Rhea. But independent of the root cause of the magnetic field and velocity perturbations near planetary bodies, their resultant Alfvén wings carry Poynting flux toward the central body.

\section{Poynting fluxes at extrasolar planets}

In this study we investigate the plasma environment at 850 extrasolar planets ${ }^{1}$ (Schneider et al. 2011). They provide a good statistical ensemble to investigate the possible electromagnetic star-planet coupling at extrasolar planets.

\subsection{Star, stellar wind, and extrasolar planet properties}

The total Poynting flux generated at the extrasolar planets can be calculated with (53) if the following properties are known: the components of the unperturbed stellar wind velocity $v_{\mathrm{sw}}$, assumed to be radially away from the star, the orbital velocity of the planet $v_{\text {orbit }}$, the radial and azimuthal component of the magnetic field, $B_{r}$ and $B_{\varphi}$ in a stellar centric reference frame, the stellar wind plasma density $\rho$, and the effective radius $R_{\text {eff }}$ of the Alfvén wings. None of these values are measured directly, yet. Instead we use the following properties provided by http://exoplanet. eu/: the extrasolar planets mass $m_{\mathrm{exo}}$ and radius $R_{\text {exo }}$, its orbital period $P_{\text {exo }}$ and radial distance $r_{\text {exo }}$ from the central star, the stellar mass $m_{\star}$, radius $R_{\star}$ and age $a_{\star}$. As described in the following paragraphs the required properties to calculate the Poynting fluxes can be estimated with this data.

In case the set of measured values on http://exoplanet . $\mathrm{eu} /$ is not complete for the extrasolar planets or their associated host stars, we proceed as follows: In case either the mass $m_{\text {exo }}$ or the radius $R_{\text {exo }}$ of one the 850 extrasolar planets are not known, we estimate the missing value assuming a mass density $\rho_{J}$ equal to Jupiter's interior. For some extrasolar planets there is no data of either the radial distance $r_{\text {exo }}$ or the orbital period $P_{\text {exo }}$ available. In these cases we calculate the missing value with Kepler's third law. If either the masses $m_{\star}$ or the radii $R_{\star}$, or both values of the stars are missing, we estimate the missing values based

1 Listed until 2012, November 14 on the website http: //exoplanet. eu/ 
Table 2. Overview and classification of the 850 extrasolar planets and their associated stars under consideration according to (a) stellar class and Alfvén Mach number and (b) applied strategies when not all stellar properties to model the stellar wind are available.

\begin{tabular}{|c|c|c|c|c|}
\hline \multirow{9}{*}{ (a) } & & & Total number & Number with $M_{\mathrm{A}}<1$ \\
\hline & spectral class: B & & 2 & 1 \\
\hline & spectral class: A & & 9 & 8 \\
\hline & spectral class: $\mathrm{F}$ & & 94 & 52 \\
\hline & spectral class: $\mathrm{G}$ & & 325 & 82 \\
\hline & spectral class: $\mathrm{K}$ & & 200 & 58 \\
\hline & spectral class: $\mathrm{M}$ & & 45 & 17 \\
\hline & spectral class: not available & & 175 & 77 \\
\hline & sum of all classes: & & 850 & 295 \\
\hline \multirow[t]{11}{*}{ (b) } & calculation of $R_{\text {exo }}$ & with $m_{\mathrm{exo}}$ and $\rho_{\mathrm{J}}$ & 548 & 99 \\
\hline & calculation of $m_{\mathrm{exo}}$ & with $R_{\mathrm{exo}}$ and $\rho_{\mathrm{J}}$ & 39 & 13 \\
\hline & calculation of $P_{\text {exo }}$ & with $r_{\mathrm{exo}}$ and $m_{\star}$ & 26 & 15 \\
\hline & calculation of $r_{\text {exo }}$ & with $P_{\text {exo }}$ and $m_{\star}$ & 58 & 27 \\
\hline & calculation of $m_{\star}$ and $R_{\star}$ & based on spectral class & 50 & 8 \\
\hline & calculation of $m_{\star}$ and $R_{\star}$ & for unknown spectral class with $\rho_{\odot}$ and $R_{\odot}$ & 17 & 14 \\
\hline & calculation of $R_{\star}$ & with $m_{\star}$ and $\rho$ based on spectral class & 134 & 28 \\
\hline & calculation of $R_{\star}$ & with $m_{\star}$ and $\rho_{\odot}$ for unknown spectral class & 28 & 2 \\
\hline & calculation of $m_{\star}$ & with $R_{\star}$ and $\rho$ based on spectral class & 1 & 0 \\
\hline & calculation of $m_{\star}$ & with $R_{\star}$ and $\rho_{\odot}$ for unknown spectral class & 9 & 6 \\
\hline & substitution of $a_{\star}$ & with $a_{\odot}$ & 304 & 100 \\
\hline
\end{tabular}

on the stars' spectral classes. In these cases we assume that the stars are main-sequence stars and use the typical values for radius, mass and thus density associated with their spectral classes as provided in Appendix G of Carroll \& Ostlie (2007). In case the spectral class is unknown in addition to an unknown stellar mass or radius, we assume the values of the sun. Furthermore we use the age $a_{\odot}$ of the sun for unknown stellar ages. In Table 2 we provide an overview of some classifications of the 850 extrasolar planets and their host stars. The table also shows how often unknown properties had to be approximated as described in this paragraph.

We estimate the stellar winds properties based on known properties of the central star and a model for the evolution of the stellar wind. Most observed extrasolar planets orbit sun like stars. Preusse et al. (2005) compare the stellar wind model by Parker (1958) and Weber \& Davis (1967) and find that the Parker model is generally adequate to describe the stellar wind properties around the known extrasolar planets (see also discussion in Sect. 4.2). The plasma and field properties near extrasolar planets which orbit very close to the central star might possibly be altered due to the local stellar field structures such as for example due to coronal loops. In our solar system, Wang \& Sheeley (1995) approximate the solar magnetic field by a potential field as far out as $2.5 R_{\odot}=0.011 \mathrm{AU}$. Outside that region the current sheet effects have to be considered and the field and the flow are described by the Parker model (Wang \& Sheeley 1995; Lockwood et al. 1999). To calculate $v_{\mathrm{sw}}, B_{\mathrm{r}}, B_{\varphi}$ and $\rho$ at the radial distance $r_{\text {exo }}$ with the Parker model, interior boundary conditions are needed. These conditions are given by the measured values $m_{\star}, R_{\star}$ and the unknown values temperature $T_{\star}$ of the corona, the magnetic field $B_{\star}$ of the star, its rotation period $P_{\star}$ and the mass flow density $F_{\star}$ at its surface. In the absence of direct measurements of the stellar corona temperatures, we assume $T_{\star} \approx 10^{6} \mathrm{~K}$ comparable to the corona temperatures of the sun. The magnetic field of the extrasolar planet hosting stars is generally unknown and thus needs to be estimated. Based on the age of the star $a_{\star}$, we estimate its rotation period after Newkirk (1980) with the empirical relationship $P_{\star} \sim\left(1+a_{\star} / \tau\right)^{0.7}$ and the constant $\tau=2.56 \times 10^{7}$ years. The magnetic field of the star $B_{\star}$ can therefore be estimated after Grießmeier et al. (2007) with the relationship $B_{\star}=B_{\odot} P_{\odot} / P_{\star}$ using values of the solar magnetic field $B_{\odot}=1.44 \times 10^{5} \mathrm{nT}$ (Preusse et al. 2006) and the solar rotation period $P_{\odot}=27$ days. Because the stellar mass flow density is generally unknown, we assume the same value as on the sun's surface $F_{\star}=2.2 \times 10^{-10} \mathrm{~kg} \mathrm{~s}^{-1} \mathrm{~m}^{-2}$ (based on a particle density of $6.6 \mathrm{~cm}^{-3}$ and a velocity of $425 \mathrm{~km} \mathrm{~s}^{-1}$ at $1 \mathrm{AU}$ from Schwenn 1990).

The orbital velocities of the planets are constrained by Kepler's third law and contribute to the relative velocity between the planets and the stellar winds through their azimuthal velocity $v_{\text {orbit }}(\operatorname{see}(5))$.

For calculating the energy fluxes generated in the interaction of stellar winds with extrasolar planets, the effective radius $R_{\mathrm{eff}}$ of the Alfvén wing is required, which in turn is controled by the planetary magnetic moment (in case it possesses one). Magnetic moments $\boldsymbol{M}_{\text {exo }}$ of extrasolar planets are not known observationally, yet, even though it is generally assumed that extrasolar planets likely possess intrinsic magnetic fields (e.g., Christensen et al. 2009). In the absence of measurements, we assume a scaling law for the dipole magnetic moment of convection-driven planetary dynamos according to Eq. (23) of Olson \& Christensen (2006), which we scale to values of Jupiter and find

$M_{\text {exo }}=M_{J}\left(\frac{R_{\text {exo }}}{R_{J}}\right)^{10 / 3}$,

with the magnetic moment of Jupiter $M_{\mathrm{J}}=1.56 \times 10^{27} \mathrm{Am}^{2}$ (Connerney et al. 1982) and the equatorial radius of Jupiter $R_{\mathrm{J}}=$ $71492 \mathrm{~km}$ (Lindal et al. 1981). We assume that the magnetic moment and the stellar wind magnetic field are perpendicular based on the majority of the configurations in the solar system, so that $\Theta_{M}=90^{\circ}$. We also assume that the extrasolar planets possess ionospheres which lead to a strong plasma interaction with $\bar{\alpha}=1$. 

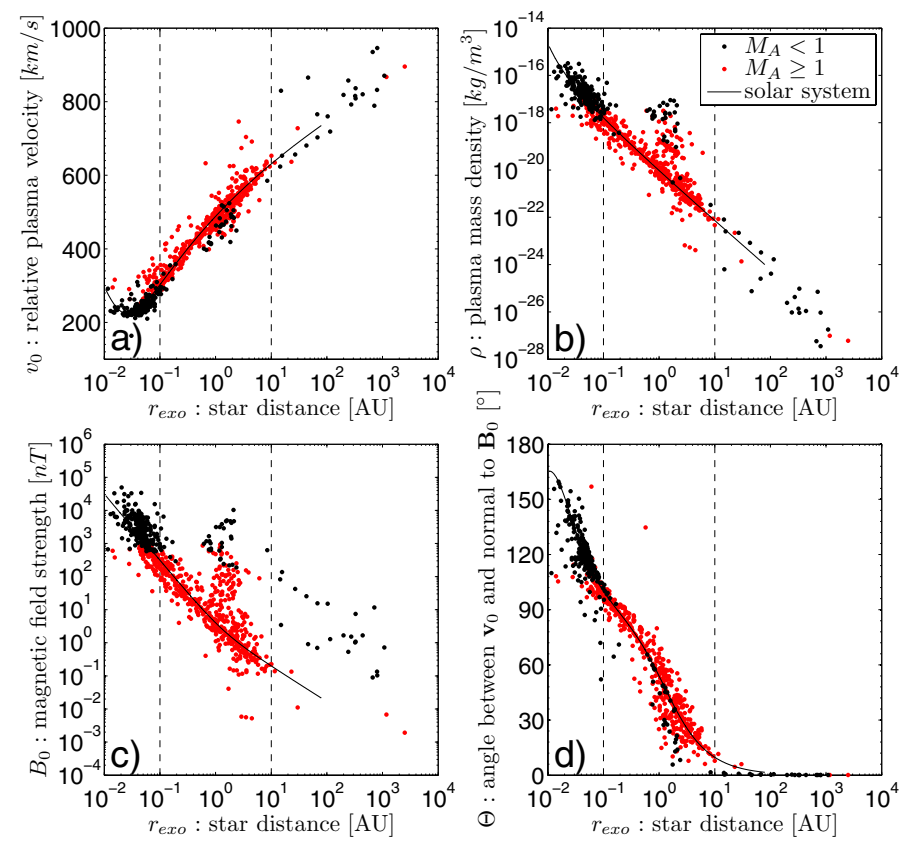

Fig. 9. Solar wind properties upstream of 850 extrasolar planets under consideration as a function of radial distance from the central star. a) Relative plasma velocity between stellar wind and planet; b) plasma density; c) magnetic field strength; d) angle $\Theta$, i.e., deviation of magnetic field and relative plasma velocity from being perpendicular. Black dots are for Alfvén Mach numbers $M_{\mathrm{A}}$ smaller than one and red dots are for $M_{\mathrm{A}}>1$ (see also Fig. 10).

\subsection{Results of statistical study}

In Fig. 9, we display the undisturbed stellar wind properties near the 850 extrasolar planets under consideration as function of radial distance from their central stars. This figure includes the relative plasma velocity $v_{0}$ between the extrasolar planet and the stellar wind (Fig. 9a), the plasma density $\rho$ (Fig. 9b), the magnetic field strength $B_{0}$ (Fig. 9c), and the angle $\Theta$ between the normal to the magnetic field and the relative flow velocity (Fig. 9d) as a function of radial distance. Note that the Poynting flux is symmetric with respect to $\Theta=90^{\circ}$ (see also discussion in Sect. 2.2). The thin solid line displays the properties of the solar wind in the solar system. The black dots are for cases in which the stellar wind is sub-Alfvénic, i.e. $M_{\mathrm{A}}<1$, and the red dots for $M_{\mathrm{A}}>1$. In Fig. 10 we display the Alfvén Mach numbers $M_{\mathrm{A}}$ near the 850 extrasolar planets as a function of radial distance calculated from the stellar wind properties shown in Fig. 9.

We find that the Alfvén Mach number distribution has a roughly similar dependency as expected from the Mach number in the solar wind shown as thin solid line in Fig. 10. The reason is that most known extrasolar planets orbit solar like stars. As expected $M_{\mathrm{A}}<1$ is more likely to be met when an extrasolar planet is close to the star. We separate the extrasolar planets in three groups: an inner region close to the stars $\left(r_{\text {exo }}<0.1 \mathrm{AU}\right)$, an intermediate region $\left(0.1 \mathrm{AU} \leq r_{\text {exo }}<10 \mathrm{AU}\right)$, and an outer region $\left(10 \mathrm{AU} \leq r_{\mathrm{exo}}\right)$. These regions are indicated as vertical lines in Figs. 9-11. Overall we find that 295 of the 850 planets under consideration are exposed to a stellar wind plasma with Alfvén Mach number $M_{\mathrm{A}}$ less than one. We note that due to observational and model uncertainties and due natural temporal variations some extrasolar planets which are close to the $M_{\mathrm{A}}=1$ line might shift from sub-Alfvénic to super-Alfvénic interaction and vice versa. In the inner region the probability for $M_{\mathrm{A}}<1$ is the highest and is met by 234 out of 303 extrasolar planets. In the intermediate region it is fulfilled for 39 out of 519 and in the outer region for 22 out of 28 extrasolar planets.

For some extrasolar planets, $M_{\mathrm{A}}$ lies significantly above the solar wind reference line, which contributes to cases with $M_{\mathrm{A}}>1$. Most of the stars in the inner and intermediate region, where this is the case, belong to the stellar class $\mathrm{M}$, which are color coded in red in Fig. 10. M type stars have small radii and masses compared to the sun and thus generate large stellar wind velocities within the Parker model. The smaller radii of $\mathrm{M}$ type stars also generate weaker stellar magnetic fields and thus smaller Alfvén velocities. Both effects contribute, on average, to larger values of $M_{\mathrm{A}}$ compared to the solar case. The opposite holds for young-type stars. Extrasolar planets in the intermediate region with $M_{\mathrm{A}}<1$ are possible in the opposite case, i.e. for large and young stars, which have a large rotation rate and thus a strong magnetic field (see Sect. 4.1). Yet observed extrasolar planets in the outer regions mostly orbit very young stars. Thus nearly all extrasolar planets in this region are, at first glance surprisingly, subject to a sub-Alfvénic plasma interaction. The currently most distant planet with $M_{\mathrm{A}}<1$ is according to our model SR 12 AB c. As will be discussed below, many of these extrasolar planets, however, still cannot connect magnetically to the host star due to unsuitable directions of their Alfvén wings.

The total Poynting flux generated at each extrasolar planet where the interaction is sub-Alfvénic is shown in Fig. 11 with the color code displaying the stellar class of the central star. The values of the total Poynting flux vary by many orders of magnitude and have a general tendency to decrease with radial distance. For some of the extrasolar planets, our calculated stellar wind properties are in a parameter space such that condition (56) is violated. In this case, we calculate a lower limit by decreasing the interaction strength $\bar{\alpha}$ until condition (56) is met. These cases are displayed as circles in Fig. 11.

Large Poynting fluxes are observed for extrasolar planets very close to the central stars and at radial distances around $1 \mathrm{AU}$, while the lowest Poynting fluxes are achieved for planets around 0.1 AU. Within the studied ensemble of extra solar planets, no Poynting flux arrives at the central star for $r_{\text {exo }} \gtrsim$ a few AU for reasons to be discussed in the following paragraph. According to (55) the total Poynting flux is proportional $B_{0}^{3} \rho^{-1} \cos ^{2} \Theta$. The product of $B_{0}^{3} \rho^{-1}$ decreases by roughly 15 orders of magnitude according to Fig. 9 within 0.01 and $1000 \mathrm{AU}$, which generates the decreasing trend with radial distance. But the angular deviations $\Theta$ from the flow being perpendicular to the stellar wind magnetic field also play a pronounced role (Fig. 9d). Near 0.1-0.3 AU we find a corridor with $\Theta \approx 90^{\circ}$ where the flow and the magnetic field are nearly aligned with each other (Fig. 9d). In this corridor, $\cos ^{2} \Theta \approx 0$ and the motional electric field vanishes. Thus no or only very little Poynting flux is generated in the interaction. This effect is responsible for the local minimum of the total Poynting fluxes in the corridor between 0.1-0.3 AU displayed in Fig. 11. The effect of a vanishing motional electric field in this corridor was first noticed in Zarka et al. (2001) and then applied in Zarka (2007) to calculations of the energy fluxes convected onto extrasolar planets. Particular large Poynting fluxes are also observed for some extrasolar planets near $1 \mathrm{AU}$. This region is outside the "corridor" region and the flow direction and the magnetic field direction are again sufficiently different to generate large Poynting fluxes. The extrasolar planets in this region with the largest Poynting fluxes orbit the largest stars of our ensemble of stars with an interaction with $M_{\mathrm{A}}<1$. These extrasolar planets 


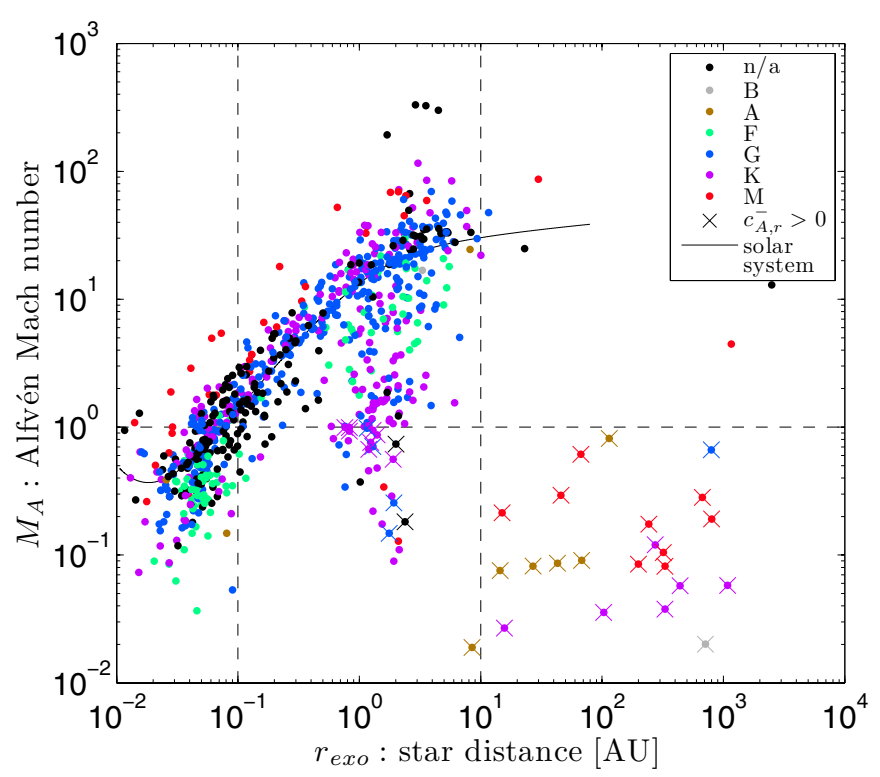

Fig. 10. Alfvén Mach number $M_{\mathrm{A}}$ as a function of radial distance for the sample of 850 planets considered in this study. The spectral classes of the extrasolar planet hosting stars are color coded. The thin solid line represents $M_{\mathrm{A}}$ in the solar wind of our solar system. Planets with $M_{\mathrm{A}}<1$ whose wings still do not couple to the central star are marked with crosses (see text).

are also relative large in size and thus generate according to the scaling law of (59) after Olson \& Christensen (2006) large planetary magnetic moments.

An Alfvén Mach number less than one is, however, not a sufficient condition to establish an electromagnetic coupling between an extrasolar planet and its central star. In several cases, marked with crosses in Fig. 10, $M_{\mathrm{A}}$ is less than one, but the directions of both wings point away from the star. This is the case when the stellar wind's azimuthal magnetic field component is large, i.e. the Parker magnetic field spiral is sufficiently wound up. Then the two Alfvén wings are directed away from the star. This is for example the case for HD $11977 \mathrm{~b}$ as shown with the two red wings $\left(\boldsymbol{c}_{\mathrm{A}}^{+}\right.$and $\left.\boldsymbol{c}_{\mathrm{A}}^{-}\right)$in Fig. 12. In the same figure, we place a hypothetical planet at closer radial distance to demonstrate the case when one wing points toward the star (green dotted line) and thus electromagnetically couples to the star. For the wing that couples to the star a phase lag between the exoplanet and its "footprint" on the star is generated as discussed, e.g., in Preusse et al. (2006) or Kopp et al. (2011). In case where $M_{\mathrm{A}}<1$ and both wings point away from the star, the Poynting fluxes calculated with the full nonlinear expression (53) consistently lead to negative values and are thus not shown in Fig. 11.

Alfvén waves travel parallel to the magnetic field in the restframe of the unperturbed plasma. The exact criterion that at least one wing points toward the star thus reads

$\left.\boldsymbol{v}_{0}\right|_{r}<\left.\boldsymbol{v}_{\mathrm{A}}\right|_{r}$,

i.e., the radial component of the relative velocity between the stellar wind and the planet $\left.\boldsymbol{v}_{0}\right|_{r}$ needs to be slower than the radial component of the Alfvén velocity $\left.\boldsymbol{v}_{\mathrm{A}}\right|_{r}=\left.\boldsymbol{B}_{0}\right|_{r} / \sqrt{\mu_{0} \rho}$. If the stellar wind is azimuthally symmetric, then the wing which points toward (away from) the star will (not) connect to the star. For example, if the magnetic field $\boldsymbol{B}_{0}$ is perpendicular to the radial stellar wind velocity $\boldsymbol{v}_{\mathrm{sw}}$, then the radial component of the Alfvén velocity is zero and both wings always point away from the star (if $v_{\mathrm{sw}}$ is directed away from the star).

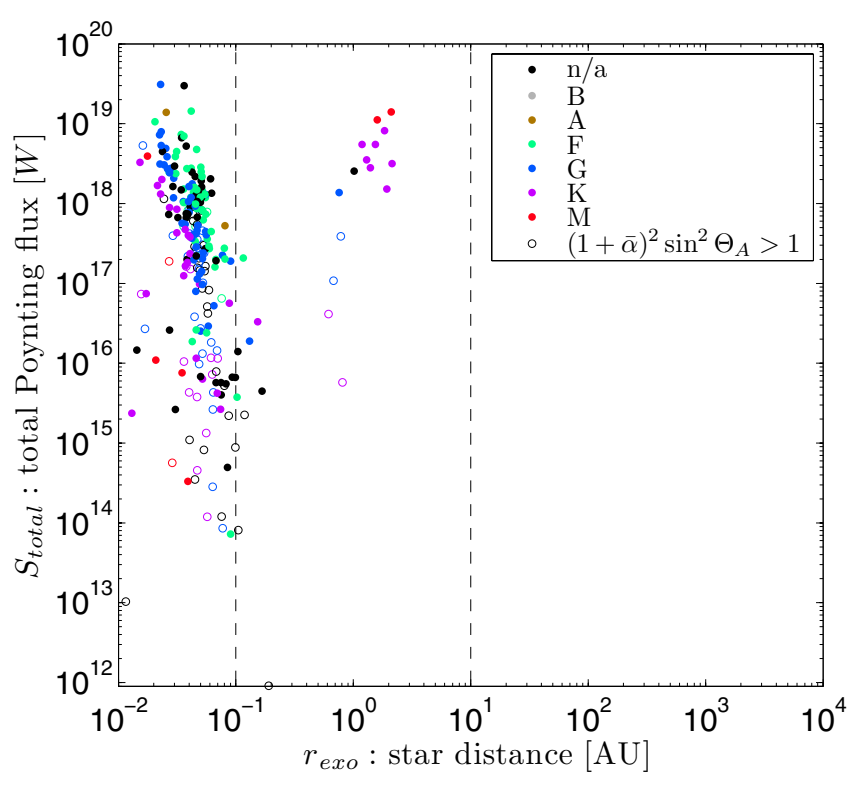

Fig. 11. Total Poynting fluxes carried in Alfvén wings as a function of distance from the star for all 258 planets with sub-Alfvénic plasma interaction which connect to the central star. The classes of the central stars are color coded. Planets marked with a cross generate two Alfvén wings, which are both pointed away from the star and thus do not connect to the central star. For extrasolar planets where condition (56) is met, we accordingly lower the interaction strength $\bar{\alpha}$ and thus provide a lower limit for the Poynting flux shown as circles.

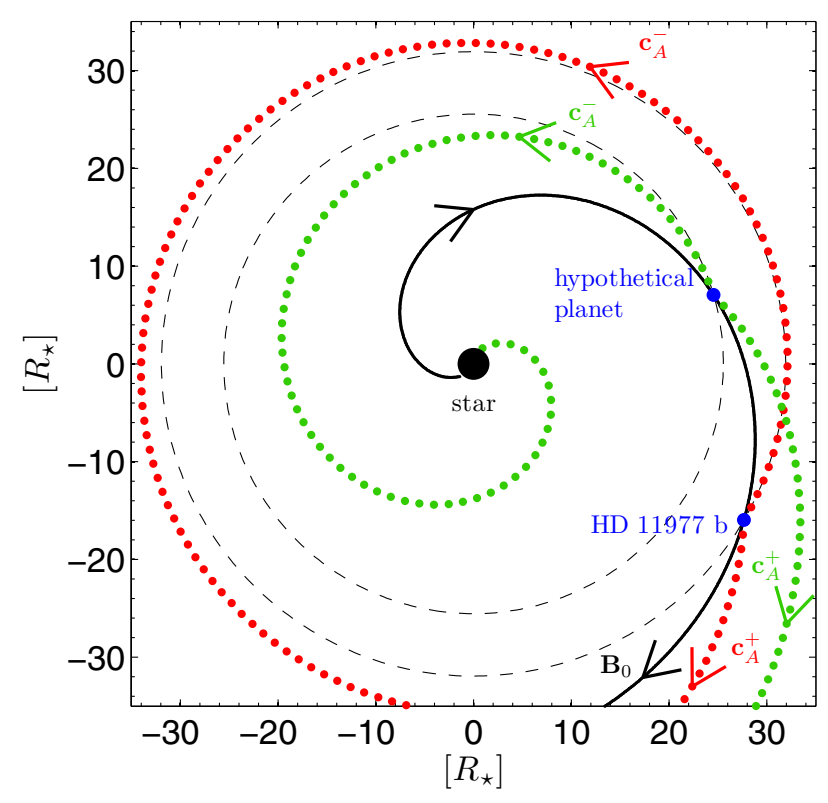

Fig. 12. Alfvén wings for HD 11977 b and a hypothetical planet at closer radial distance. Both Alfvén wings (in red) generated at HD $11977 \mathrm{~b}$ point away from the star and thus do not connect to it. A hypothetical planet at closer radial distance displays one wing that points toward the star and one wing away from it (shown in green). The black solid line displays a magnetic field line in the stellar wind and the dashed lines the orbital distances of the two planets.

Note, that for extrasolar planets the central body is generally not located purely in the upstream direction of the relative flow because the relative velocity between the stellar wind and the planet generally possesses an azimuthal component due to the orbital velocity of the planet. Also note that condition (60) 
Table 3. Overview of measured, estimated and calculated properties leading to the total Poynting flux of HD $179949 \mathrm{~b}$.

\begin{tabular}{|c|c|c|c|c|c|}
\hline \multicolumn{2}{|c|}{$\begin{array}{l}\text { Measured properties } \\
\text { (http://exoplanets.eu/) }\end{array}$} & \multicolumn{2}{|c|}{$\begin{array}{l}\begin{array}{l}\text { Estimated properties similar to sun system } \\
\text { (see Sect. 4.1) }\end{array} \\
\end{array}$} & \multicolumn{2}{|c|}{$\begin{array}{l}\text { Interaction properties } \\
\text { (see Sect. 4.1) }\end{array}$} \\
\hline$m_{\mathrm{exo}}$ & $0.95 m_{\mathrm{J}}$ & $T_{\star} \approx T_{\odot}$ & $10^{6} \mathrm{~K}$ & $v_{\mathrm{sw}}$ & $162.8 \mathrm{~km} \mathrm{~s}^{-1}$ \\
\hline$P_{\text {exo }}$ & 3.09 days & $F_{\star} \approx F_{\odot}$ & $2.17 \times 10^{-10} \mathrm{~kg} \mathrm{~s}^{-1} \mathrm{~m}^{-2}$ & $v_{\text {orbit }}$ & $158.3 \mathrm{~km} \mathrm{~s}^{-1}$ \\
\hline$r_{\mathrm{exo}}$ & $0.045 \mathrm{AU}$ & $B_{\star} \approx B_{\odot} P_{\odot} / P_{\star}$ & $4.31 \times 10^{5} \mathrm{nT}$ & $B_{r}$ & $6511 \mathrm{nT}$ \\
\hline$m_{\star}$ & $1.28 m_{\odot}$ & & & $B_{\varphi}$ & $-2175 \mathrm{nT}$ \\
\hline$R_{\star}$ & $1.19 R_{\odot}$ & & & $\rho$ & $2.02 \times 10^{-17} \mathrm{~kg} \mathrm{~m}^{-3}$ \\
\hline$a_{\star}$ & $2.05 \mathrm{Gyr}$ & & & & \\
\hline $\begin{array}{l}R_{\text {exo }} \\
P_{\star}\end{array}$ & $\begin{array}{c}\text { n.a. } \\
9 \text { days }^{a}\end{array}$ & $R_{\mathrm{exo}} \approx\left(3 m_{\mathrm{exo}} /\left(4 \pi \rho_{\mathrm{J}}\right)\right)^{1 / 3}$ & $0.983 R_{\mathrm{J}}$ & $M_{\text {exo }}$ & $1.47 \times 10^{27} \mathrm{~A} \mathrm{~m}^{2}$ \\
\hline
\end{tabular}

Notes. ${ }^{(a)}$ Shkolnik et al. (2005); Preusse et al. (2006).

can be fulfilled if the interaction is super-Alfvénic, i.e. $M_{\mathrm{A}}>1$. However, the theoretical model of the Alfvén wings applied in this paper is designed for sub-Alfvénic interaction only as the nature of the interaction changes for $M_{\mathrm{A}}>1$.

The geometrical situation when both wings point away from the star is similar to the sub-Alfvénic interaction at the satellites of Jupiter and Saturn. At these satellites the magnetic field and the plasma flow are nearly perpendicular and thus the waves do not travel upstream, but are inclined toward the downstream direction. However, the central body, i.e. Jupiter and Saturn, are not upstream of the satellites, but nearly perpendicular to the flow direction and there is no (or only very little) relative velocity between the satellites and their central planets (in the radial and latitudinal direction).

The five extrasolar planets with the largest Poynting fluxes in decreasing order are WASP-12 b $\left(3.1 \times 10^{19} \mathrm{~W}\right)$, KOI-13 b $\left(3.0 \times 10^{19} \mathrm{~W}\right)$, WASP-78 b $\left(1.4 \times 10^{19} \mathrm{~W}\right)$, HD $208527 \mathrm{~b}$ $\left(1.4 \times 10^{19} \mathrm{~W}\right)$, WASP-33 b $\left(1.4 \times 10^{19} \mathrm{~W}\right)$. As supplementary information we provide the plasma properties and the calculated Poynting fluxes for all 850 extrasolar planets considered in this study in a Table available at the CDS. Our estimated stellar wind properties might be helpful for other studies of the interaction of extrasolar planets with their surrounding plasma environment. This table includes the names of the extrasolar planets, their distances from the host stars, the Kepler velocities of the extrasolar planets, the stellar wind velocities calculated with the Parker model, the radial and azimuthal components of the stellar wind magnetic fields, the stellar wind mass densities, the Alfvén Mach numbers, the total Poynting fluxes generated by the interaction, and details about the nature of the possible interactions and our calculations. The stellar wind properties provided in the online table are estimated near the vicinity of the extrasolar planets. Note, in many cases stellar properties are unknown and had to be estimated. Similarly, the existences, strengths and orientations of the magnetic moments of extrasolar planets are unknown. We used a scaling law to derive their magnetic moments which enter into our calculations of the total Poynting fluxes (see Sect. 4.1).

We also investigate if the kinetic or thermal energy flux can be positive toward the star with expressions (41) to (46) and find this is the case for only 8 of the extrasolar planets under consideration. These extrasolar planets are very close to the central star and the stellar wind magnetic field is still nearly radial. The resulting kinetic and thermal energy fluxes are, however, at least three orders of magnitude smaller compared to the respective Poynting fluxes and thus can be neglected.

In our calculations, the corona temperatures and the mass fluxes of the stars are assumed parameters based on typical values of our sun. We also studied how the number of extrasolar planets subject to sub-Alfvénic plasma flow and their resultant total Poynting fluxes vary if we, for example, increase the corona temperature to $2 \times 10^{6} \mathrm{~K}$ or modify the coronal mass outflow by a factor of two. Even though individual values change, we find that the overall statistical distributions shown in Fig. 10 and Fig. 11 do not change qualitatively. For a factor of two larger/smaller mass fluxes, for example, the average Alfvén Mach number and the average total Poynting flux are somewhat enhanced/lowered, but still stay within the range of $10^{13}$ to $10^{20} \mathrm{~W}$. According to Preusse et al. (2005) for a corona temperature of $2 \times 10^{6} \mathrm{~K}$, the differences between the stellar wind model after Parker (1958) and Weber \& Davis (1967) become very small even for very fast rotating stars.

We note that not all of the energy in the Poynting flux generated at the extrasolar planets and traveling toward the central star will actually reach the central star. Part of the wave energy will be reflected/filamented in inhomogeneous plasma densities and magnetic fields or converted into heat and particle acceleration. Similar processes also occur to the Alfvén waves generated at the satellites of Jupiter and Saturn (Wright \& Schwartz 1989; Chust et al. 2005; Jacobsen et al. 2007; Hess et al. 2010a).

The statistical study of the Poynting fluxes shows largely varying energy fluxes by many orders of magnitude. Only a few of the extrasolar planets generate energy fluxes on the order of $10^{19} \mathrm{~W}$ while the majority of the extrasolar planets generates fluxes many orders of magnitude smaller. These large variations are even seen within close radial distances of 0.1 AU. This could be a natural explanation why statistical studies of planet star interactions such as performed by Poppenhaeger et al. (2010) and Poppenhaeger \& Schmitt (2011) do not find a statistically significant correlation between stellar emission and close-in extrasolar planets. Therefore strong and thus directly observable planet star coupling might only be possible for a few individual targets.

\subsection{Poynting flux generated by HD $179949 \mathrm{~b}$}

Shkolnik et al. (2005) measure the excess energy flux in the chromospheric emission ascribed to magnetic planet star coupling for HD 179949 b to be $\sim 10^{20} \mathrm{~W}$. This value can be quantitatively compared with the calculations from Sect. 4.2. Table 3 gives an overview of the measured and estimated values to calculate the Poynting flux.

In the vicinity of HD 179949 b we calculate for the unperturbed stellar wind a relative velocity of $227 \mathrm{~km} \mathrm{~s}^{-1}$, a plasma density of $2.02 \times 10^{-17} \mathrm{~kg} \mathrm{~m}^{-3}$, a magnetic field strength of $6870 \mathrm{nT}$, and the deviation of the flow from being perpendicular to the magnetic field of $\Theta=115.7^{\circ}$. If we assume a dipole moment of HD $179949 \mathrm{~b}$ according to the scaling law in Eq. (59) 

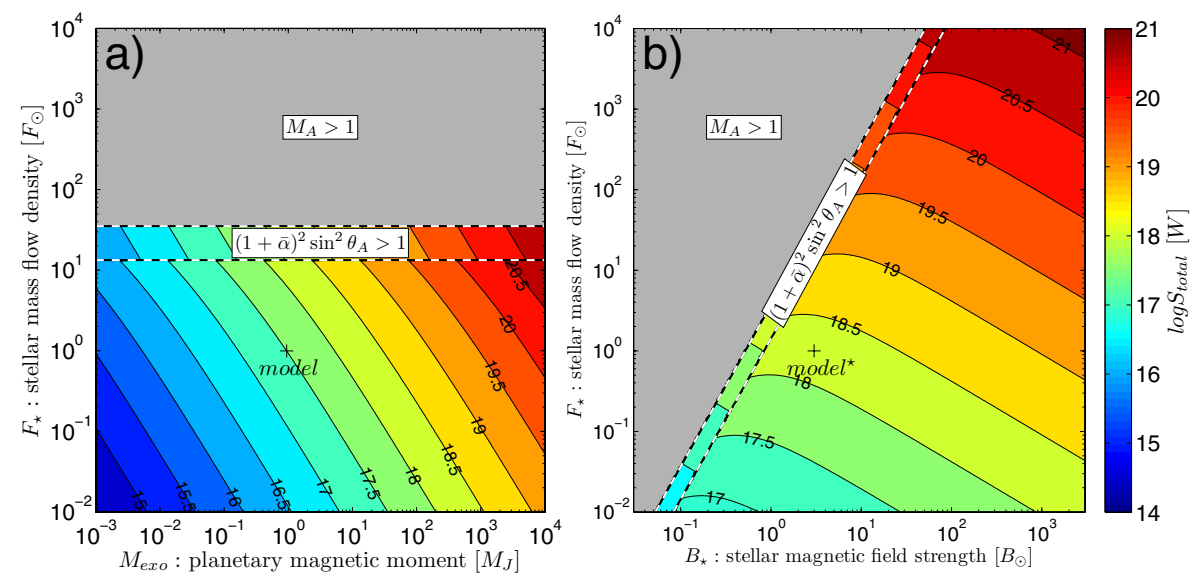

Fig. 13. Total Poynting flux generated at HD 179949 b as a function of a) stellar mass flow density $F_{\star}$ and planetary magnetic moment $M_{\text {exo }}$ and b) stellar mass flow density $F_{\star}$ and field strength $B_{\star}$ with $M_{\text {exo }}=12 M_{\mathrm{J}}$. The reference model with $M_{\text {exo }}=0.94 M_{\mathrm{J}}$ is calculated with scaling law (59) and labeled model; the reference model with $M_{\text {exo }}=12 M_{\mathrm{J}}$ is labeled model $l^{\star}$.

of $M_{\text {exo }}=1.47 \times 10^{27} \mathrm{~A} \mathrm{~m}^{2}$ which corresponds to a surface magnetic field of $4.25 \times 10^{5} \mathrm{nT}$, we find that HD $179949 \mathrm{~b}$ generates a total Poynting flux of $3.36 \times 10^{17} \mathrm{~W}$. Here we assumed that the dipole moment is perpendicular to the stellar magnetic field $\left(\Theta_{M}=90^{\circ}\right)$. Our value of the total Poynting flux is a factor of 2.5 higher compared to the estimate in Lanza (2009), but still a factor of $\sim 300$ smaller compared to the energies quoted in Shkolnik et al. (2005).

Because there are uncertainties of the used values, we explore parameter space to see what conditions are required to achieve a Poynting flux on the order of $10^{20} \mathrm{~W}$. To calculate the total Poynting flux for HD 179949 b, we made assumptions on four properties: the corona temperature $T_{\star}$, the mass flow density $F_{\star}$ and magnetic field $B_{\star}$ of the central star are not available without assuming solar system values and/or scaling laws. As the existence and possible strengths of intrinsic magnetic fields of extrasolar planets have not been observationally demonstrated, the magnetic dipole moment $M_{\text {exo }}$ of HD 179949 b is also entirely uncertain. We thus investigate the influence of these four parameters. A guide for the dependence of some of the parameters can be achieved by using the simplified expression for the Poynting flux (55) and the extension of the Alfvén wing (57) which leads to

$S_{\text {total }} \propto B_{0}^{1 / 3} M_{\text {exo }}^{2 / 3} \rho^{1 / 2} v^{2}$.

Note that the values in this expression describe the properties near the planet and not at the star.

Our chosen stellar corona temperature already renders Poynting fluxes near its maximum value. A factor 10 lower value would cause a much weaker total energy flux of $\sim 4 \times 10^{12} \mathrm{~W}$. Assuming higher corona temperatures would basically lower the Poynting flux, too.

In Fig. 13a we show the total Poynting flux as function of stellar mass flow density $F_{\star}$ and planetary magnetic dipole moment $M_{\text {exo }}$. For high mass flow densities there is a large parameter space where $M_{\mathrm{A}}$ turns larger than 1 . The high plasma density $\rho$, resulting from high $F_{\star}$, decreases the Alfvén velocity and prohibits an energy flux directed to the central star. In a small interval of $F_{\star}$ condition (56) is fulfilled. Here we approximate $S_{\text {total }}$ with expression (55). For comparability the values of our standard model are marked with a cross. In logarithmic presentation $S_{\text {total }}$ is a linear function of the magnetic dipole moment as the Poynting flux scales with $M_{\text {exo }}^{2 / 3}$.

The surface magnetic field strength of HD $179949 \mathrm{~b}$ would need to be a factor of 4000 stronger than at Jupiter to explain a Poynting flux of $10^{20} \mathrm{~W}$. Christensen et al. (2009) argue that extrasolar planets can have a magnetic field with a surface strength of 5-12 times the field strength of Jupiter. Using a factor of 12 the Poynting flux can not reach an order of $10^{20} \mathrm{~W}$ even with the assumption of a higher mass flow density. If $F_{\star}$ is a factor 10 higher than on the sun, the planetary magnetic moment still has to be a factor 500 stronger to produce a Poynting flux consistent with the energy fluxes quoted in Shkolnik et al. (2005). Because this is a rather unrealistic magnetic moment we look for other options to simulate the measurements. Varying $B_{\star}$ in our calculations linearly changes the stellar magnetic field near the extrasolar planet $B_{0}$ and yields a weakly increasing energy flux with stronger magnetic fields (see Fig. 13 and Eq. (61)). The influence of $B_{\star}$ is too weak to achieve an order of $10^{20} \mathrm{~W}$. The magnetic field would need to be a factor 100 stronger to reach only a slightly higher energy flux of $\sim 10^{18} \mathrm{~W}$. Note, our expressions for the Poynting fluxes even yield a weaker dependence on $B_{0}$ compared to previous estimates with more simplified expressions of the local energetics (e.g., Lanza 2009). Figure 13b shows the dependency of the Poynting flux from stellar mass flow density $F_{\star}$ and magnetic field $B_{\star}$. To avoid exotic planetary properties, we set the magnetic dipole moment of HD179949 b equal to 12 times the field strength of Jupiter for this calculation. Next to this assumption $F_{\star}$ and $B_{\star}$ both still have to be at least a factor 100 higher, which is rather unlikely. Zarka (2007) and Lanza (2009) also come to similar conclusions that the observed fluxes by Shkolnik et al. (2005) require large or unrealistically large planetary and stellar fields, respectively, if the only energy source is the energy of the local interaction.

We also note that additionally not all the energy in the Poynting fluxes will be fully delivered to the star as the Alfvén waves experience filamentation and probably also scattering in a possibly turbulent stellar wind.

If the energy flux in the star planet interaction at HD $179949 \mathrm{~b}$ is indeed as strong as estimated by Shkolnik et al. (2005), then energy releases in coronal loops as suggested by Lanza (2009) might be an additional mean to produce the required energy. These releases, however, will be triggered and the associated planet star coupling will be energetically supplemented by the Alfvén waves launched at the planets calculated in this paper.

\section{Conclusions and discussion}

In this work we derive expressions for the Poynting flux in subAlfvénic plasma interaction, which can be applied to planetary moons in the outer solar system, but also to the coupling between extrasolar planets and their central stars. The expressions are calculated based on full nonlinear solutions of shear Alfvén waves 
within the framework of MHD (Neubauer 1980). We also provide simplified expressions for the total Poynting flux for small Alfvén Mach numbers (see Eqs. (54) or (55)), which are "user friendly" applicable, compared to our full expressions. Our expressions provide values for the Poynting flux generated near the planetary obstacles and thus likely present upper limits for the energy fluxes deposited into the central bodies since a fraction of the wave energy might be partially reflected or dissipated while traveling to the central bodies.

The flux values calculated in this work are consistent with footprint brightnesses observed at Jupiter's moons Io, Europa, and Ganymede and Saturn's moon Enceladus. We also model how the Poynting fluxes generated at the Galilean satellites vary as a function of system III in Jupiter's magnetosphere.

We perform a statistical analysis on the existence and strength of magnetic planet star coupling based on all extrasolar planets known until 2012 November 14. We find that 295 of 850 extrasolar planets are embedded in a sub-Alfvénic, i.e. in an $M_{\mathrm{A}}<1$ plasma environment. This is, however, only a necessary but not a sufficient condition that the Alfvénic interaction can transport energy upstream to the star. An additional requirement is that one of the two Alfvén wings generated in the interaction has a directional component toward the central star. This reduces the number of extrasolar planets within our ensemble which can magnetically couple to the star to 258 . We find that the total Poynting fluxes generated at extrasolar planets in generally strongly decrease as a function of the planets distance from the central star within the first 0.1 AU. A local minimum of the flux is found near 0.1 AU because the local stellar wind magnetic field and the relative plasma velocities are often nearly parallel at theses distances (Zarka 2007).

Our modeled energy flux generated by HD $179949 \mathrm{~b}$ is more than two orders of magnitude smaller compared to the $\sim 10^{20} \mathrm{~W}$ derived by Shkolnik et al. (2005). Only with rather exotically strong magnetic fields of the star HD 179949 and its companion values as derived by Shkolnik et al. (2005) can be achieved. The maximum modeled energy fluxes of our statistical study are on the order of $10^{19} \mathrm{~W}$ and thus still an order of magnitude smaller than the values derived by Shkolnik et al. (2005). If indeed observable planet star interaction requires energy fluxes on the order of $10^{20} \mathrm{~W}$, then further energy sources are likely necessary. For example, the energy flux in the Alfvén waves originating at the extrasolar planets might trigger magnetic energy releases in coronal loops as suggested by Lanza (2009). In this case, still a threshold energy flux will be needed to trigger the additional release of coronal energy.

Our statistical analysis shows that the energy fluxes within the subset of extrasolar planets with $M_{\mathrm{A}}<1$ vary by many orders of magnitudes even for the close-in extrasolar planets within 0.1 AU. Our findings might provide a natural explanation why statistical studies based on observations by Poppenhaeger et al. (2010) and Poppenhaeger \& Schmitt (2011) find no statistical correlation between X-ray and optical luminosity of the stars and close-in extrasolar planets, but do not rule out the existence of electromagnetic planet star interaction for individual extrasolar planets.

Acknowledgements. J.S. appreciates helpful discussions and comments by Peter Olson during his sabbatical stay at the Johns Hopkins University. J.S. also thanks Bertrand Bonfond and Sebastien Hess for helpful comments on the original manuscript. We thank Mario Seufert for providing values of his magnetic field model and Sven Jacobsen for providing density profiles of the Io plasma torus. Part of this work was supported by the German Deutsche Forschungsgemeinschaft, DFG with the Schwerpunktsprogramm Planetary Magnetism.

\section{References}

Acuña, M. H., Neubauer, F. M., \& Ness, N. F. 1981, J. Geophys. Res., 86, 8513 Bagenal, F. 1994, J. Geophys. Res., 99, 11043

Bagenal, F., \& Delamere, P. A. 2011, J. Geophys. Res., 116, A05209

Bigg, E. K. 1964, Nature, 203, 1008

Bonfond, B. 2012, in Auroral Phenomenology and Magnetospheric Processes: Earth and Other Planets, eds. A. Keiling, et al. (Washington, D.C.: Geophys. Monogr. Ser.), 197, 133

Bonfond, B., Gérard, J.-C., Grodent, D., \& Saur, J. 2007, Geophys. Res. Lett., 340, L06201

Bonfond, B., Grodent, D., Gérard, J., et al. 2008, Geophys. Res. Lett., 35, L05107

Bonfond, B., S., H., Gérard, J., et al. 2013, Planet. Space Sci., submitted

Carroll, B. W., \& Ostlie, D. A. 2007, in An introduction to modern astrophysics (San Francisco: Perason, Addion Wesley)

Chané, E., Saur, J., Neubauer, F. M., Raeder, J., \& Poedts, S. 2012, J. Geophys. Res., 117, 9217

Christensen, U. R., Holzwarth, V., \& Reiners, A. 2009, Nature, 457, 167

Chust, T., Roux, A., Kurth, W. S., et al. 2005, Plant. Space Sci., 53, 395

Clarke, J. T. Ballester, G. E., Trauger, J., et al. 1996, Science, 274, 404

Clarke, J. T., Ballester, G. E., Trauger, J., et al. 1998, J. Geophys. Res., 103, 20217

Clarke, J. T., Ajello, J., Ballester, G. E., et al. 2002, Nature, 415, 997

Clarke, J. T. Benoit, C., Bradford, D., et al. 2011, in Magnetospheres of the Outer Planets, Boston, Massachusetts

Cohen, O., Drake, J. J., Kashyap, V. L., et al. 2009, ApJ, 704, L85

Connerney, J., Acuña, M. H., \& Ness, N. F. 1982, J. Geophys. Res., 87, 3623

Connerney, J. E. P., Baron, R., Satoh, T., \& Owen, T. 1993, Science, 262, 1035

Cuntz, M., Saar, S. H., \& Musielak, Z. E. 2000, ApJ, 533, L151

Dougherty, M. K., Khurana, K. K., Neubauer, F. M., et al. 2006, Science, 311, 1406

Drell, S. D., Foley, H. M., \& Ruderman, M. A. 1965, J. Geophys. Res., 70, 3131

Elsässer, W. 1950, Phys. Rev., 79, 183

Frank, L. A., Paterson, W. R., Ackerson, K. L., et al. 1996, Science, 274, 394

Gérard, J.-C., Saglam, A., Grodent, D., \& Clarke, J. 2006, J. Geophys. Res., 111, A04202

Goertz, C. K. 1980, J. Geophys. Res., 85, 2949

Goldreich, P., \& Lynden-Bell, D. 1969, ApJ, 156, 59

Grießmeier, J., Stadelmann, A., Penz, T., et al. 2004, A\&A, 425, 753

Grießmeier, J., Motschmann, U., Mann, G., \& Rucker, H. O. 2005, A\&A, 437, 717

Grießmeier, J., Zarka, P., \& Spreeuw, H. 2007, A\&A, 475, 359

Grodent, D., Gérard, J., Gustin, J., et al. 2006, Geophys. Res. Lett., 33, L6201

Grodent, D., Bonfond, B., Radioti, A., et al. 2009, J. Geophys. Res., 114, 07212

Gustin, J., Bonfond, B., Grodent, D., \& Gérard, J.-C. 2012, J. Geophys. Res., 117,7316

Hess, S. L. G., \& Zarka, P. 2011, A\&A, 531, A29

Hess, S. L. G., Delamere, P., Dols, V., Bonfond, B., \& Swift, D. 2010a, J. Geophys. Res., 115, 06205

Hess, S., Bonfond, B., Delamere, P. A., et al. 2010b, AGU Fall Meeting Abstracts, C1775

Hess, S. L. G., Delamere, P. A., Dols, V., \& Ray, L. C. 2011a, J. Geophys. Res. Space Phys., 116, A01202

Hess, S. L. G., Delamere, P. A., Bagenal, F., Schneider, N., \& Steffl, A. J. 2011b, J. Geophys. Res., 116, 11215

Hess, S. L. G., et al. 2013, Plant. Space Sci., submitted

Ip, W.-H., Kopp, A., \& Hu, J. 2004, ApJ, 602, L53

Jacobsen, S. 2011, Dissertation, Institut für Geophysik und Meteorologie, Universität zu Köln

Jacobsen, S., Neubauer, F. M., Saur, J., \& Schilling, N. 2007, Geophys. Res. Lett., 34, 10202

Jacobsen, S., Saur, J., Neubauer, F. M., et al. 2010, J. Geophys. Res., 115, 4205

Jia, X., Walker, R., Kivelson, M., Khurana, K., \& Linker, J. 2009, J. Geophys. Res., 114, 09209

Jia, Y.-D., Russell, C. T., Khurana, K. K., et al. 2010, J. Geophys. Res., 115, 04214

Khurana, K. K., Dougherty, M. K., Russell, C. T., \& Leisner, J. S. 2007, J. Geophys. Res., 112, A8

Kivelson, M. G., Khurana, K. K., Russell, C. T., et al. 1996a, Nature, 384, 537

Kivelson, M. G., Khurana, K. K., Walker, R. J., et al. 1996b, Science, 273, 337

Kivelson, M. G., Bagenal, F., Neubauer, F. M., et al. 2004, in Jupiter, ed. F. Bagenal (University of Colorado: Cambridge Univ. Press), 513

Kopp, A., Schilp, S., \& Preusse, S. 2011, ApJ, 729, 116

Kriegel, H., Simon, S., Müller, J., et al. 2009, Planet. Space Sci., 57, 2113

Kriegel, H., Simon, S., Motschmann, U., et al. 2011, J. Geophys. Res., 116, 10223 
Lanza, A. F. 2008, A\&A, 487, 1163

Lanza, A. F. 2009, A\&A, 505, 339

Li, J., Ferrario, L., \& Wickramasinghe, D. 1998, ApJ, 503, L151

Lindal, G. F., Wood, G. E., Levy, G. S., et al. 1981, J. Geophys. Res., 86, 8721

Lipatov, A. S., Motschmann, U., Bagdonat, T., \& Grießmeier, J. 2005, Planet. Space Sci., 53, 423

Lockwood, M., Stamper, R., \& Wild, M. N. 1999, Nature, 399, 437

Neubauer, F. M. 1980, J. Geophys. Res., 85, 1171

Neubauer, F. M. 1998, J. Geophys. Res., 103, 19843

Newkirk, Jr., G. 1980, in The Ancient Sun: Fossil Record in the Earth, Moon and

Meteorites, eds. R. O. Pepin, J. A. Eddy, \& R. B. Merrill, 293

Olson, P., \& Christensen, U. R. 2006, Earth Planet. Sci. Lett., 250, 561

Parker, E. N. 1958, ApJ, 128, 664

Piddington, J. H., \& Drake, J. F. 1968, Nature, 217, 935

Poppenhaeger, K., \& Schmitt, J. H. M. M. 2011, ApJ, 735, 59

Poppenhaeger, K., Robrade, J., \& Schmitt, J. 2010, A\&A, 515, A98

Poppenhaeger, K., Lenz, L. F., Reiners, A., Schmitt, J. H. M. M., \& Shkolnik, E. 2011, A\&A, 528, A58

Prangé, R., Rego, D., Southwood, D., et al. 1996, Nature, 379, 323

Preusse, S., Kopp, A., Büchner, J., \& Motschmann, U. 2005, A\&A, 434, 1191

Preusse, S., Kopp, A., Büchner, J., \& Motschmann, U. 2006, A\&A, 460, 317

Preusse, S., Kopp, A., Büchner, J., \& Motschmann, U. 2007, Plant. Space Sci., 55,589

Pryor, W. R., Rymer, A. M., Mitchell, D. G., et al. 2011, Nature, 472, 331

Roussos, E., Krupp, N., Armstrong, T. P., et al. 2008, Geophys. Res. Lett., 352, L22106

Santolík, O., Gurnett, D. A., Jones, G. H., et al. 2011, Geophys. Res. Lett., 38, 19204

Saur, J. 2004, J. Geophys. Res., 109, A01210

Saur, J., \& Strobel, D. F. 2005, ApJ, 620, L115

Saur, J., Strobel, D. F., \& Neubauer, F. M. 1998, J. Geophys. Res., 103, 19947

Saur, J., Neubauer, F. M., Strobel, D. F., \& Summers, M. E. 1999, J. Geophys. Res., 104, 25105

Saur, J., Neubauer, F. M., Strobel, D. F., \& Summers, M. E. 2002, J. Geophys. Res., 107, 1422

Saur, J., Neubauer, F. M., \& Schilling, N. 2007, J. Geophys. Res., 112, A11209
Saur, J., Schilling, N., Neubauer, F. M., et al. 2008, Geophys. Res. Lett., 35, L20105

Scharf, C. A. 2010, ApJ, 722, 1547

Schilling, N. 2006, Dissertation, Institut für Geophysik und Meteorologie der Universität zu Köln

Schneider, J., Dedieu, C., Le Sidaner, P., Savalle, R., \& Zolotukhin, I. 2011, ArXiv e-prints

Schwenn, R. 1990, in Physics of the Inner Heliosphere I, eds. R. Schwenn, \& E. Marsch (Berlin: Springer), 99

Seufert, M., Saur, J., \& Neubauer, F. M. 2011, Icarus, 214, 477

Shkolnik, E., Walker, G. A. H., \& Bohlender, D. A. 2003, ApJ, 597, 1092

Shkolnik, E., Walker, G. A. H., Bohlender, D. A., Gu, P., \& Kürster, M. 2005, ApJ, 622, 1075

Shkolnik, E., Bohlender, D. A., Walker, G. A. H., \& Collier Cameron, A. 2008, ApJ, 676, 628

Simon, S., Saur, J., Neubauer, F. M., Motschmann, U., \& Dougherty, M. K. 2009, Geophys. Res. Lett., 360, L04108

Simon, S., Saur, J., Kriegel, H., et al. 2011a, J. Geophys. Res., 116, A04221

Simon, S., Saur, J., Neubauer, F. M., Wennmacher, A., \& Dougherty, M. K. 2011b, Geophys. Res. Lett., 381, L15102

Simon, S., Kriegel, H., Saur, J., et al. 2012, J. Geophys. Res., 117, 7211

Strobel, D. F., Saur, J., Feldman, P. D., \& McGrath, M. A. 2002, ApJ, 581, L51

Teolis, B. D., Jones, G. H., Miles, P. F., et al. 2010, Science, 330, 1813

Tokar, R. Johnson, R. E., Hill, T. W., et al. 2006, Science, 311, 1409

Wang, Y.-M., \& Sheeley, Jr., N. R. 1995, ApJ, 447, L143

Wannawichian, S., Clarke, J. T., \& Nichols, J. D. 2010, J. Geophys. Res., 115, A02206

Weber, E. J., \& Davis, Jr., L. 1967, ApJ, 148, 217

Willes, A. J., \& Wu, K. 2004, MNRAS, 348, 285

Willes, A. J., \& Wu, K. 2005, A\&A, 432, 1091

Wright, A. N., \& Schwartz, S. J. 1989, J. Geophys. Res., 94, 3749

Zarka, P. 1998, J. Geophys. Res., 103, 20159

Zarka, P. 2006, in Planetary Radio Emission VI, eds. H. O. Rucker, W. S. Kurth, \& G. Mann (Austria: Austrian Academy of Sciences Press), 543

Zarka, P. 2007, Plant. Space Sci., 55, 598

Zarka, P., Treumann, R. A., Ryabov, B. P., \& Ryabov, V. B. 2001, Ap\&SS, 277,293 